\title{
重氮化合物转化中的氮基团保留反应研究进展
}

\author{
邱頔*, ${ }^{\text {邱孟龙 }}{ }^{a}$ 马戎 ${ }^{a} \quad$ 张艳 $^{b}$ 王剑波 ${ }^{b}$ \\ $\left({ }^{a}\right.$ 天津师范大学化学学院 天津市功能分子结构与性能重点实验室 \\ 无机一有机杂化功能材料化学教育部重点实验室 天津 300387) \\ $\left(^{b}\right.$ 北京大学化学与分子工程学院 生物有机与分子工程教育部重点实验室 北京分子科学国家实验室 北京 100871)
}

\begin{abstract}
摘要 重氮化合物是一类非常重要的有机合成中间体, 它在有机合成化学以及药物设计研发、化学生物学、材料化学 等领域具有重要的应用价值. 传统的重氮化合物的转化反应类型包括了 Wolff 重排, 经由过渡金属卡宾或者类卡宾中 间体的插入反应，催化的环丙烷化反应，以及近年来发展的过渡金属催化的经由卡宾中间体的交叉偶联反应等. 重氮 化合物除了发生作为卡宾前体的经典反应之外, 它们还可以经由氮基团保留的转化过程, 在目标分子中保留重氮基团 或者其它含氮原子的官能团. 该种策略提供了一种高效而选择性地构筑含氮功能分子、尤其是官能化的氮杂环的合成 途径. 其中, 不对称的 $\mathrm{C}-\mathrm{N}$ 键的选择性构筑, 以及不对称的氮杂环分子的组装, 仍然具有重要的合成价值和重大的挑 战意义. 本篇综述根据反应的机理和类型，将这部分研究工作分为六部分内容进行介绍.
\end{abstract}

关键词 重氮化合物; 有机合成; 氮杂环; 环加成

\section{Nitrogen Group Retaining Reaction in the Transformation of Diazo Compounds

\author{
Qiu, $\mathrm{Di}^{*, a} \quad$ Qiu, Menglong $^{a} \quad$ Ma, Rong $^{a} \quad$ Zhang, Yan $^{b} \quad$ Wang, Jianbo $^{b}$ \\ ( ${ }^{a}$ Tianjin Key Laboratory of Structure and Performance for Functional Molecules, Key Laboratory of Inorganic-Organic \\ Hybrid Functional Materials Chemistry of Ministry of Education, College of Chemistry, \\ Tianjin Normal University, Tianjin 300387) \\ Engineering of Ministry of Education, College of Chemistry, Peking University, Beijing 100871)
} \\ ( ${ }^{b}$ Beijing National Laboratory of Molecular Sciences (BNLMS) and Key Laboratory of Bioorganic Chemistry and Molecular}

\begin{abstract}
Diazo compounds represent a type of very important synthetic intermediates, which demonstrate wide applications in organic synthesis, continuous-in-flow technology, polymer synthesis, medicinal chemistry, chemical biology, material science and many other fields. On the other hand, diazo intermediates can be easily prepared from commercial available substrates through facile transformations, such as base-promoted decomposition of $N$-tosylhydrazones, diazo-transfer reaction, diazotization of alkyl amines, oxidation of hydrazones, decomposition of $N$-nitroso compounds. Traditional transformations of diazo compounds include nucleophilic addition/substitution by using diazo compounds as the nucleophiles, ylide type reactions, dimerization or olefination, Wolff rearrangement, transition-metal-carbene or carbenoid mediated $\mathrm{X}-\mathrm{H}$ insertion reactions, catalytic cyclopropanations or cyclopropenations, and the recently developed transition-metal-catalyzed carbenoid cross-coupling reactions. In addition to these classic reactions, the diazo compounds also undergo nitrogen group retaining reactions, in which the diazo moiety is incorporated into the nitrogen-containing moiety in the target molecules. This strategy has provided an efficient and selective synthetic approach towards nitrogen atom containing functional molecules, especially for the synthesis of various $\mathrm{N}$-heterocyclic compounds. Among them, the enantioselective $\mathrm{C}-\mathrm{N}$ bond forming reaction as well as the asymmetric $N$-heterocyclic scaffold construction has important synthetic value and remains great challenge to the organic chemists. Thus, nitrogen component retaining reactions of diazo compounds has opened up a superior avenue in organic synthesis. Considering about the significant importance and the great growth in the past decade of this area, this review article will focus on the nitrogen group retaining reaction of diazo compounds. According to the reaction mechanism of these transformations, this review will be divided into the following parts: diazo compounds as nucleophiles, diazo compounds as 1,3-dipoles in cycloaddition reaction, diazo compounds as electrophiles, intramolecular reactions of vinyldiazo compounds, reduction reaction, and miscellaneous transformation. We hope that this review will corroborate the practical use of this research area as a convenient and valuable synthetic strategy.
\end{abstract}

Keywords diazo compound; organic synthesis; $N$-heterocycle; cycloaddition

\footnotetext{
* E-mail: qiudi@pku.edu.cn; Tel.: 13811114725

Received March 29, 2016; published April 26, 2016.

Project supported by the scientific research funding of Tianjin Normal University (No. 5RL138).

项目受天津师范大学引进人才人事专项资助(No. 5RL138).
} 


\section{1 引言}

重氮化合物是一类非常重要的有机合成中间体，它 不仅在有机合成化学领域, 而且在药物设计研发、化学 生物学、材料化学、流动相反应、高分子化学以及天然 产物全合成等方面具有重要的应用价值 ${ }^{[1,2]}$. 从原料合 成的角度上，重氮中间体可以通过磺酰腙在碱性条件下 的分解、重氮转移反应、脂肪胺的重氮化、腙的氧化、 $N$-亚硝基化合物的分解等手段来快速地制备 ${ }^{[3]}$. 另一方 面, 由于重氮化合物其独特的结构, 它们可以参与到多 种多样的有机反应中去, 用来选择性地合成高度官能化 的目标分子(Scheme 1).

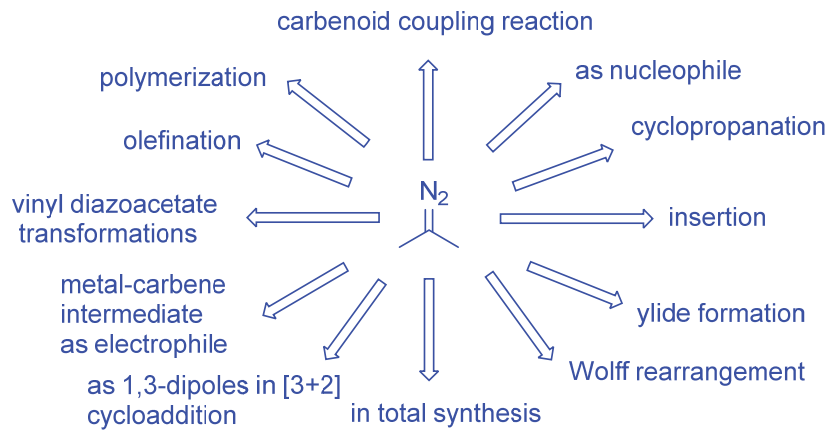

图式 1 重氮化合物的典型反应类型

Scheme 1 Typical reaction types of diazo compounds

在重氮化合物的转化过程中, 过渡金属催化的反应 是一类重要的途径 ${ }^{[4]}$ : 其中, 既包括了传统的经由过渡 金属卡宾或者类卡宾中间体的插入反应, 可以高效地构 筑碳一碳键以及碳一杂原子键; 以及催化的环丙烷化和环 丙烯化反应，立体选择性地构筑三元环骨架; 也包括了 近年来被人们广泛关注的过渡金属催化的经由卡宾中 间体的交叉偶联反应, 它经过了转移插入途径, 提供了 一种新颖的反应类型和合成功能分子的途径. 此外, 重 氮化合物还可以经由叶立德类型的转化过程, 或者 Wolff 重排等反应类型，来合成目标分子.

除了以上介绍的反应之外, 重氮化合物还可以发生 氮基团保留的转化过程, 在目标分子中保留重氮基团或 者其它含氮原子的官能团. 相比于经由过渡金属卡宾或 者类卡宾的反应途径, 这样一类典型的转化策略, 实现 了一个或者多个氮组分的保留, 提供了一种高效而选择 性地构筑含氮功能分子, 尤其是官能化的氮杂环的合成 途径. 传统的重氮保留反应, 例如重氮的二聚过程, 可 以得到二嗪衍生物. 近年来, 立体专一性的 $\mathrm{C}-\mathrm{N}$ 键的 选择性构筑, 以及不对称的氮杂环分子的组装, 仍然具 有重要的合成价值和重大的挑战意义.

考虑到含氮化合物和含氮芳杂环在有机合成、药物 研发、化学生物学等领域的重要性, 总结和归纳重氮化 合物的氮基团保留的反应, 将具有重要的理论和实际的 意义. 本文将重点关注近些年来发展的重氮化合物反应
中氮基团保留的反应，并且根据反应的机理类型进行分 类归纳，旨在总结和分析该类反应的特点以及面临的挑 战，为进一步解决这些问题提供策略和思路.

\section{2 重氮化合物作为亲核试剂的反应}

从结构的角度上，重氮化合物中，和重氮基团连接 的碳原子带有部分的负电荷, 具有一定的亲核性, 可以 发生亲核加成或者取代反应 ${ }^{[5,6]}$. 此外, 双羰基的重氮化 合物在一定的条件下可以生成烯醇式衍生的 $\alpha$-羰基重 氮中间体, 可以在烯醇位点发生亲核加成反应(Scheme 2). 这些转化过程从合成的角度上, 可以作为取代的重 氮化合物的合成方法，用以制备官能化的重氮中间体. 然而另一方面，重氮基团并没有真正参与到反应中; 因 此本文仅通过举例描述每种反应类型，对于这部分内容 进行简单的介绍.
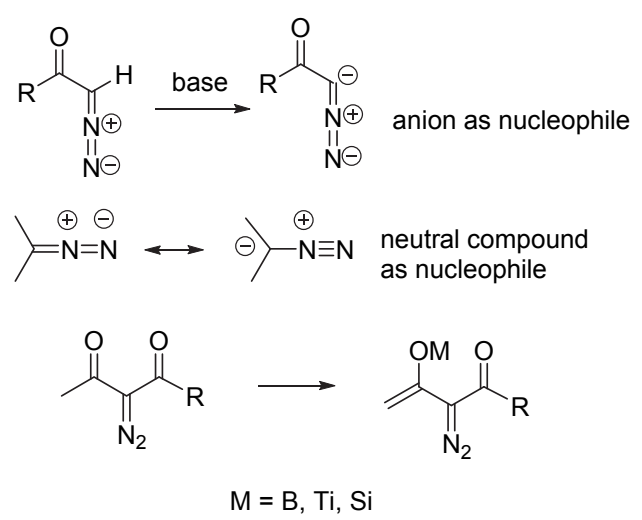

图式 2 重氮化合物或者烯醇重氮作为亲核试剂的示例

Scheme 2 General feature of diazo compounds and diazo enolates compounds as nucleophiles

重氮化合物在一定的条件下，可以和羰基化合物发 生加成反应，得到取代的 $\beta$-羟基- $\alpha$-重氮羰基化合物. 从 机理的角度上，这是一种羟醛缩合类型的反应. 2003 年， 王剑波课题组 ${ }^{[7]}$ 发展了首例重氮酯和醛发生的 aldol 类 型加成反应，以中等较高的对映选择性得到了 $\beta$-羟基 $-\alpha$-重氮羰基化合物. 该反应采用了 $\mathrm{Zr}(\mathrm{IV})-\mathrm{BINOL}$ 作为 手性催化体系(Scheme 3). 尽管反应的产率和 $e e$ 值仍有 待提高, 但该催化体系首次将重氮化合物作为亲核试剂 的 aldol 反应与不对称催化策略相结合, 为化学家们提 供了新的思路与合成途径.

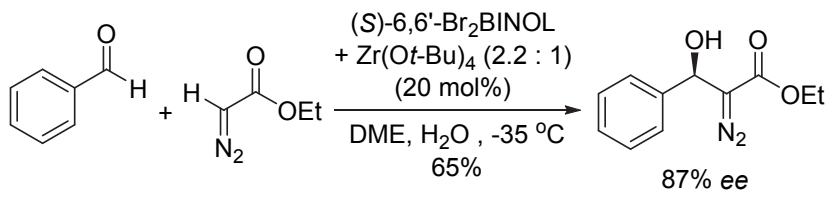

图式 3 Zr-BINOL 催化的重氮酯和醛的不对称 aldol 反应 Scheme 3 Asymmetric aldol condensation catalyzed by $\mathrm{Zr}(\mathrm{IV})-\mathrm{BINOL}$ system

近年来另一个具有代表性的例子, 是在 Nishida 
等 ${ }^{[8]}$ 发展的辛可尼定衍生物 1 作为手性助剂的催化条件 下，重氮酯和醛发生的立体选择性的加成反应(Scheme 4). 在较低的温度下, 该反应能够以中等至较高的对映 选择性制备 aldol 反应产物, 实现了重氮基团保留的同 时, 碳一碳键的选择性构建.<smiles>CCCOC(=O)C=[NH2+]</smiles>

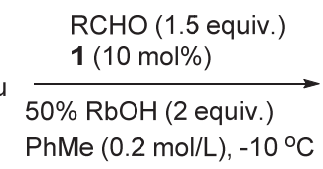<smiles>[R]C(O)C(=N[18O][Ba])C(=O)OCC</smiles>

$\mathrm{R}=$ Alkyl, Aryl

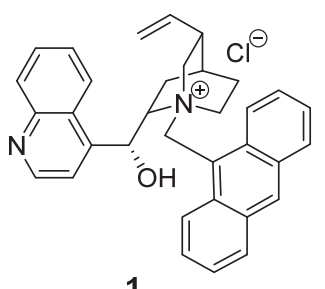

图式 4 辛可尼定盐催化的重氮化合物的不对称羟醀缩合反应

Scheme 4 Asymmetric aldol condensation by using cinchonidinium salt

2008 年, 硅胶负载的四甲基胍衍生物被开发作为 非均相催化剂, 用以促进重氮乙酸乙酯和醛的羟醛缩合 反应 ${ }^{[9 a]}$. 该反应不仅能够以很好的产率获得加成产物, 并且催化剂能够很好地回收再利用. 此外, 离子液体也 被开发为可再生的催化体系, 实现了水相的重氮化合物 的 aldol 反应 ${ }^{[9 b]}$.

2012 年, Trost 等 ${ }^{[10]}$ 发展了镁催化的重氮乙酸乙酯 和多种类型的醛的不对称 aldol 反应(Scheme 5). 该反应 采用了 ProPhenol 作为手性助剂, 反应的途径涉及到了 双核镁中间体的生成. 从合成的角度上看, 一系列取代 的 $\beta$-差圤基- $\alpha$-重氮酯能够以很好的产率和对映选择性被 制备. 并且, 通过后续串联的氧化反应和亲核加成过程, 该类产物可以进一步被转化为含有相邻手性中心的邻 二醇衍生物.<smiles>CCOC(=O)C(=[N+]=[N-])[C@@H](O)c1ccccc1</smiles>

$92 \%, 95 \%$ ee

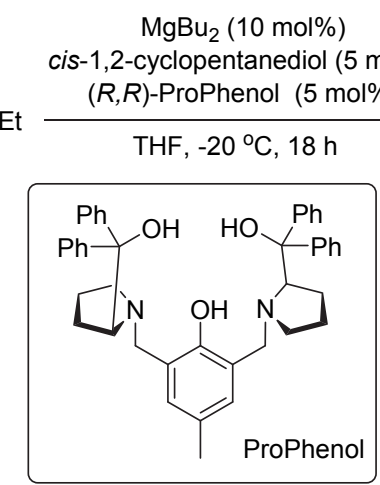

图式 $5 \mathrm{Mg}$-ProPhenol 催化的重氮乙酸乙酯和醛的不对称 aldol 反应 Scheme 5 Mg-ProPhenol-catalyzed asymmetric aldol condensation

重氮化合物也可以在 Lewis 酸或者碱的作用下, 和 亚胺发生加成反应, 得到类似Mannich 反应的加成产物. 其中, 重氮基团得到了保留. 其中的代表性反应, 例如
重氮酯在手性磷酸 2 或者手性联萗二羧酸 $\mathbf{3}$ 的催化条件 下，和芳香醛的亚胺衍生物发生不对称加成反应。该方 法能够以较高的对映选择性得到 Mannich 加成产物 (Scheme 6) $^{[11,12]}$.

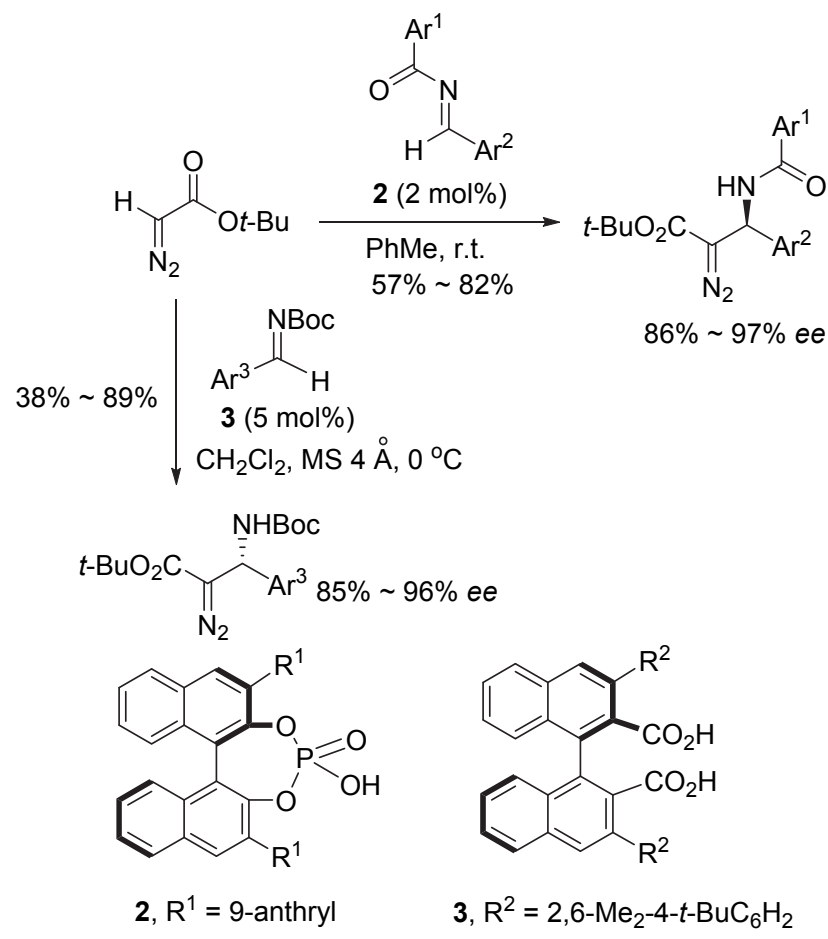

图式 6 手性联菜磷酸 2 或手性羧酸 3 催化的重氮化合物的不对称 Mannich 反应

Scheme 6 Asymmetric Mannich addition by using a chiral binaphthol monophosphoric acid or chiral dicarboxylic acid

Maruoka 等 ${ }^{[13,14]}$ 进一步利用上述的手性 Brønsted 酸 3 作为催化剂, 实现了重氮化合物和芳香醛衍生的 $N$-Boc 亚胺的不对称 Mannich 反应. 该反应具有良好的 底物普适性和对映选择性. 在温和的条件下, $\alpha$-重氮酯、 重氮磷酸酯以及重氮砜均能以较好的产率来立体选择 性地得到加成产物(Scheme 7).

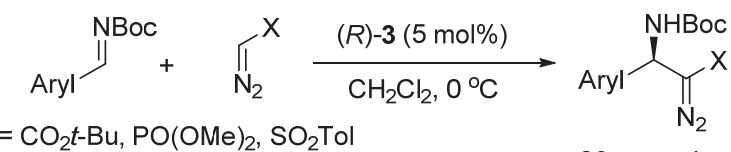

$$
\begin{aligned}
& 29 \text { examples } \\
& 38 \% \sim 89 \% \text { yields } \\
& 87 \% \sim 96 \% \text { ee }
\end{aligned}
$$

图式 7 轴手性羧酸催化的重氮酯、磷酯和砜的不对称 Mannich 反应 Scheme 7 Axially chiral dicarboxylic acid catalyzed asymmetric Mannich reaction

烯醇式衍生的 $\alpha$-重氮羰基化合物, 在保留重氮基团 的同时，可以选择性地在烯醇位点发生亲核加成反应， 得到 Mukaiyama-aldol 或者 Mukaiyama-Michael 加成产 物. 目前研究的挑战主要集中在不对称碳一碳键的构筑 中. 其中, Doyle 课题组 ${ }^{[15,16]}$ 开发了氟化银和手性联二萗 膦 4 催化条件下的不对称 Mukaiyama-aldol 反应, 以中 
等至较高的产率和较好的对映选择性得到了烯醇位点 碳一碳键构筑的产物(Scheme 8).

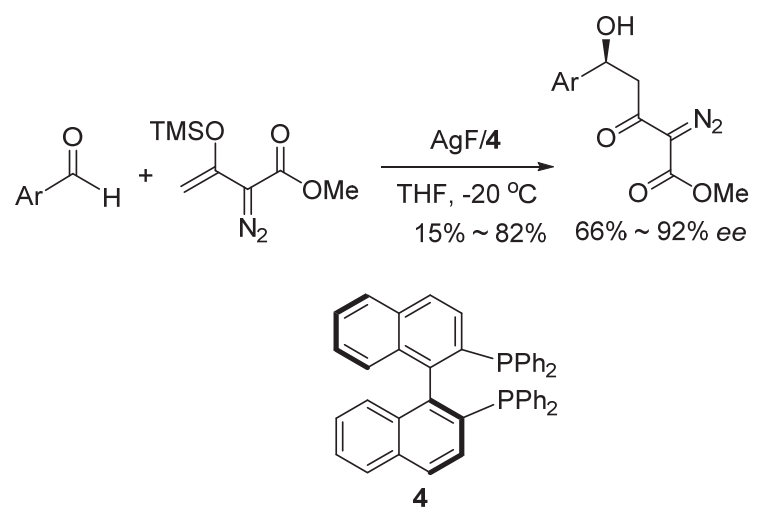

图式 8 银和联二菜膦催化的重氮烯醇硅醚和醛的 MukaiyamaMichael 加成

Scheme 8 Mukaiyama-aldol addition with silyl enol ethers catalyzed by chiral BINAP

近年来, Doyle 课题组 ${ }^{[17]}$ 进一步实现了锌催化的烯 醇式的 $\alpha$-重氮羰基化合物和取代的 $\alpha, \beta$-不饱和酮的 Mukaiyama-Michael 反应. 在三氟甲磺酸锌作为 Lewis 酸的催化条件下，官能化的重氮乙酰乙酸甲酯能够以较 高的产率被制备. 该催化体系被成功用于一锅法的重氮 乙酰乙酸酯和醛的 Mukaiyama-aldol 加成反应中 (Scheme 9) ${ }^{[18]}$. 进一步的, 通过采用二价铜催化剂和叔 丁基取代的手性噁唑啉作为配体的催化体系，高效的不 对称的 Mukaiyama-Michael 反应也被开发, 能够以很高 的对映选择性获得手性的重氮乙酰乙酸酷衍生物 ${ }^{[19]}$.

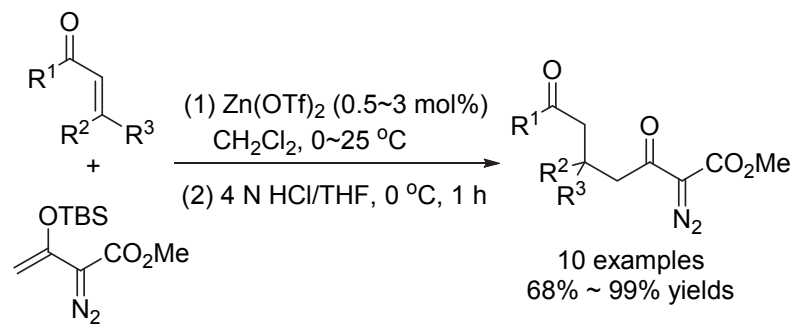

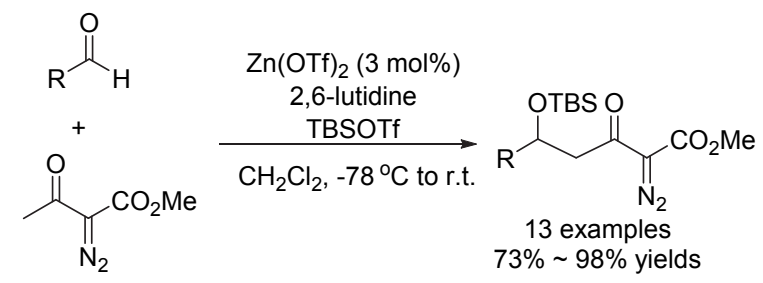

图式 9 锌催化的 Mukaiyama-Michael 反应和 Mukaiyama-aldol 反应 Scheme $9 \mathrm{Zn}(\mathrm{II})$-catalyzed Mukaiyama-Michael addition to $\alpha, \beta$-unsaturated enones and Mukaiyama-aldol reactions to aldehydes

2013 年, 手性 Lewis 碱 5 催化的 $\alpha$-重氮羰基化合物 与 Morita-Baylis-Hillman 碳酸酯作为亲电试剂的反应被 开发, 实现了烯丙位选择性的碳一碳键构筑过程(Scheme $10)^{[20]}$. 该反应经历了 $\mathrm{S}_{\mathrm{N}} 2^{\prime} / \mathrm{S}_{\mathrm{N}} 2^{\prime}$ 途径, 区域选择性和立体 专一性地合成了高度官能化的重氮化合物. 相应的产物
可以用于含氮杂环和其他功能分子的快速构筑.

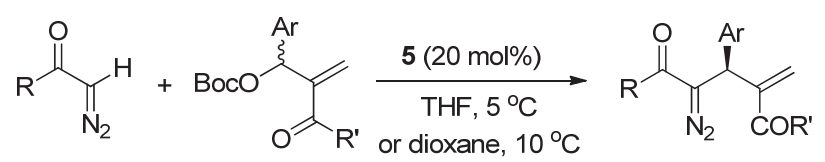

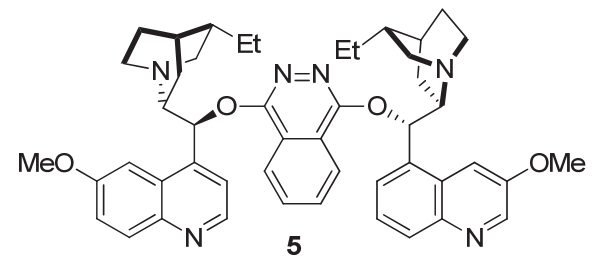

图式 10 手性 Lewis 碱 5 催化的 Morita-Baylis-Hillman 碳酸酯的烯丙 位碳-碳键构筑

Scheme 10 Chiral Lewis base-catalyzed allylic alkylation with carbonates

除了传统的重氮化合物的亲核碳原子的加成反应 之外，过渡金属催化的重氮化合物的偶联反应也进一步 被开发, 实现了重氮基团保留的碳-碳键选择性偶联反 应. 王剑波课题组 ${ }^{[21]}$ 开发了钯催化的碘化物和重氮酸 酯的直接偶联，以及在 $\mathrm{CO}$ 气氛下的三组分羰基插入偶 联反应. 此外, 该课题组还实现了 $\beta$-羰基- $\alpha$-重氮化合物 和芳基碘化物在碱性条件和钯催化剂作用下的脱乙酰 基偶联反应(Scheme 11) ${ }^{[22]}$. 这些反应提供了一种新颖 而高效的合成羰基重氮和重氮磷酸酯的直接方法. 并 且，从机理的角度，该类策略将过渡金属催化的交叉偶 联反应、重氮化学、羰基插入和脱乙酰基偶联相结合，进 一步丰富了交叉偶联反应策略的应用范围.

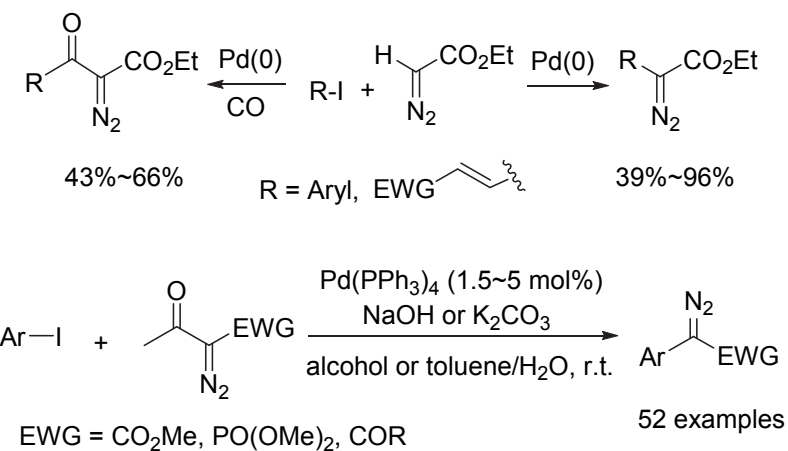

图式 11 钯催化的重氮化合物的偶联反应

Scheme 11 Pd-catalyzed cross-coupling reaction with diazo compounds

\section{3 重氮化合物作为 1,3-偶极子参与的环加成反} 应

由于重氮化合物或中间体其独特的结构，它们可以 作为高活性的 1,3-偶极子参与到环加成反应中 ${ }^{[23]}$. 根据 亲 1,3-偶极子的不同，可以将这些环加成反应分为和烯 烃的加成、炔烃的加成、联烯的加成、以及其它亲偶极 子的加成反应. 


\section{1 与烯烃的环加成反应}

一般的, 重氮化合物和贫电子的烯烃可以在无催化 剂的条件下直接发生环加成反应, 得到取代的吡唑啉. 该方法是一种传统的、底物普适性良好的 ${ }^{[24]}$ 合成官能化 的吡唑啉的通用途径(Scheme 12).

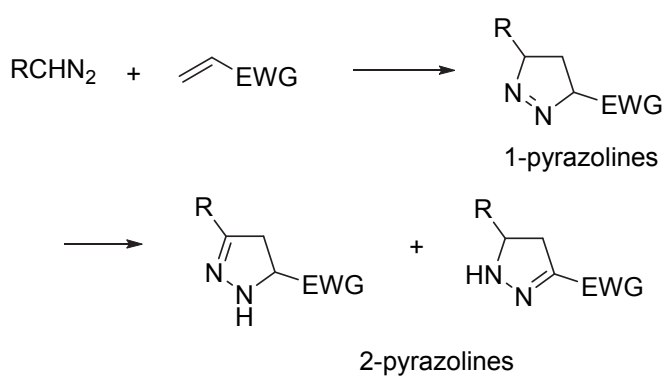

图式 12 重氮化合物与贫电子烯烃的 1,3-偶极子环加成反应

Scheme 12 General synthetic route of 1,3-dipolar cycloaddition of diazo compounds with electron-deficient alkenes

当采用重氮烷烃或者羰基重氮化合物为原料时，该 环加成反应的亲 1,3-偶极子组分底物适用性广泛, 涵盖 了不同类型的烯烃, 包括丙烯酸酯衍生物 ${ }^{[24 a \sim 24 d]}$, Morita-Baylis-Hillman 碳酸酯 ${ }^{[24 \mathrm{e}]}$, 丙烯腈 ${ }^{[24 \mathrm{~d}]}$, 丙烯酰胺 ${ }^{[24 \mathrm{~d}]}$, 戊烯二酸 ${ }^{[24 \mathrm{f}]}$, 亚砜取代的 $\alpha, \beta$-不饱和环酮 ${ }^{[24 \mathrm{~g}, \mathrm{~h}]}$, 共轭二

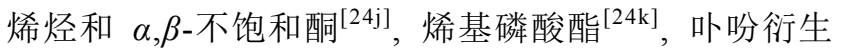

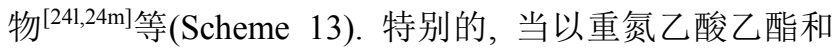
硝基取代的烯烃 ${ }^{[24 n]}$ 为反应底物时, 可以通过一锅法的 串联反应直接制备官能化的吡唑衍生物.

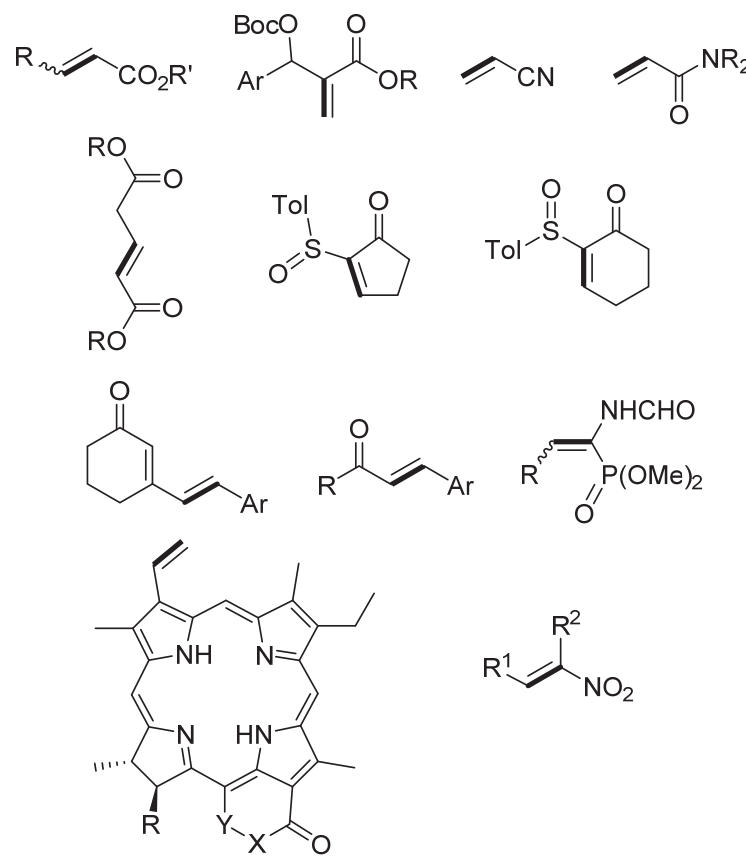

图式 13 1,3-偶极子环加成的烯烃底物普适性

Scheme 13 Scope of substrates for 1,3-dipolar cycloaddition

除了吡唑啉之外, 吡唑衍生物也可以通过该类策略 进行直接合成(Scheme 14). 在碱的参与或者催化条件 下，羰基化合物可以和重氮酯发生环加成以及后续的脱
水过程，从而生成高度官能化的吡唑骨架 ${ }^{[25]}$.
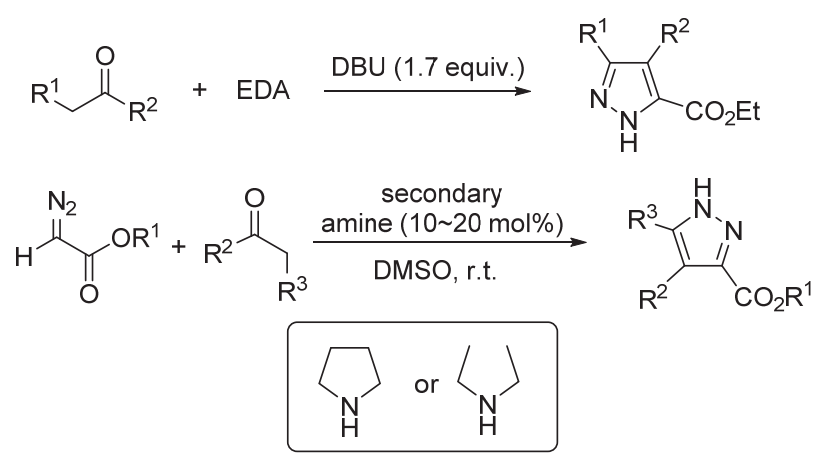

图式 14 碱参与的羰基化合物与重氮的环加成反应合成吡唑 Scheme 14 Base-mediated synthesis of pyrazoles from carbonyl compounds with diazoesters

从多氟取代的烷基胺盐酸盐出发, Mykhailiuk

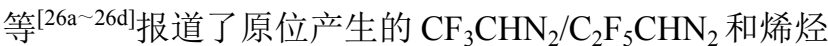
的 1,3-偶极子环加成反应，用于直接选择性地制备三氟 甲基或者五氟乙基取代的吡唑啉. 该反应需要在较低温 度下进行. 肖文精课题组 ${ }^{[26 e]}$ 发现, 对于三氟乙胺盐酸 盐产生的重氮的环加成反应，对应的产物如果在较高温 度下处理，相应的吡唑啉环会发生脱氮气过程，得到三 氟甲基取代的三元环(Scheme 15). 该反应途径提供了 一种制备三氟甲基衍生的环丙烷的补充合成策略.

$$
\begin{aligned}
& \text { Mykhailiuk 's work }
\end{aligned}
$$

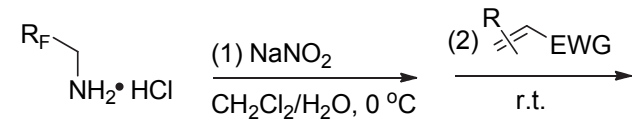

$$
\begin{aligned}
& \mathrm{R}_{\mathrm{F}}=\mathrm{CF}_{3}, \mathrm{C}_{2} \mathrm{~F}_{5}
\end{aligned}
$$

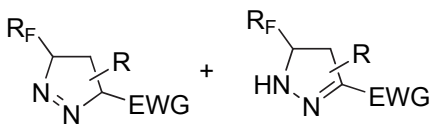

Xiao's work
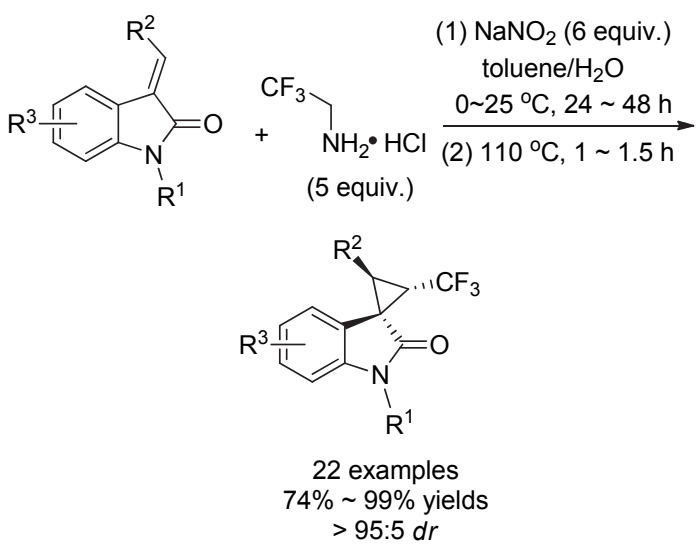

图式 15 原位产生的氟取代的重氮中间体的 1,3-偶极子环加成 Scheme 15 1,3-Dipolar cycloaddition of in situ generated fluorinated diazo intermediates and sequential ring contraction

Namboothiri 和其他课题组 ${ }^{[27}$ 开发了碱介导的贫电 
子烯烃和重氮磷酸酯和重氮磺酸酯的环加成反应, 提供 了一种高效的合成取代的吡唑衍生物的补充途径 (Scheme 16). 该反应可以高效而区域选择性地制备磷 酰基和磺酰基取代的吡唑骨架.<smiles>[R]C=C([R])[N+](C)(C)C</smiles><smiles>[R]c1[nH]nc(S(=O)(=O)[Al])c1[R]</smiles>

图式 16 贫电子烯烃与重氮磷酸酯或磺酸酯的环加成制备吡唑

Scheme 16 Synthesis of functionalized pyrazoles from Bestmann-Ohira reagent, $\alpha$-diazo- $\beta$-ketosulfone

除此之外, 官能化的手性吡唑啉的选择性合成进一 步被人们所关注 ${ }^{[28]} .2011$ 年, 镍(II)-联荎二亚胺配体体 系被开发用来催化重氮酯与贫电子烯烃的不对称 1,3-偶 极子环加成反应, 选择性地制备了含有手性中心的吡唑 啉衍生物, 并且具有良好的对映选择性(Scheme 17) ${ }^{[28 a]}$.<smiles>[R]C=C[Hg]CN1N(C(=O)C=C[R])C(=O)CC1(C)C</smiles>

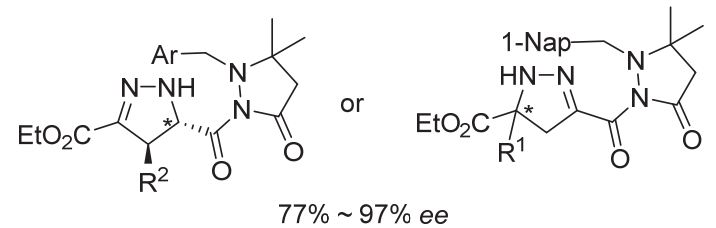

图式 17 镍催化的重氮酯的不对称 1,3-偶极子环加成反应

Scheme 17 Binaphthyldiimine-nickel-catalzyed asymmetric 1,3-dipolar cycloaddition of diazoesters

Ryu 课题组 ${ }^{[28 b, 28 c]}$ 进一步利用手性噁唑硼烷体系 6 或 7 , 以取代的重氮酯和丙烯醛或者丙烯酮衍生物为底 物, 实现了立体选择性地构筑官能化的吡唑啉. 该催化 反应具有良好的对映选择性以及较高的产率(Scheme 18).

有机小分子催化策略在合成杂环骨架中具有显著 的应用价值. 其中, 金鸡纳碱衍生物也被用来作为手性 助剂, 实现了重氮磷酸酯(Seyferth-Gilbert 试剂)与青定红 衍生的丙二腈的选择性环加成反应(Scheme 19) ${ }^{[28 d]}$. 该 反应可以高效而立体专一的合成手性吡唑啉-着基吲哚 骨架. 该不对称合成策略还可以从靛红、丙二腈和重氮 磷酸酯三组分出发, 首先经由 Knoevenagel 缩合反应及 后续的环加成反应，一锅法地来直接制备具有光活性的 目标杂环分子.

此外, 多组分以及串联的环加成反应策略也被人们

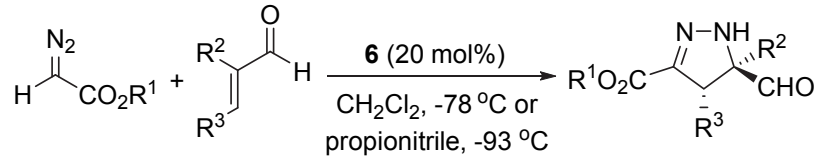<smiles>[R]c1ccccc1B1OC([Al])([Al])[C@@H]1CCC[OH2+]</smiles>

$6 \mathrm{R}=\mathrm{Me}, \mathrm{H}$

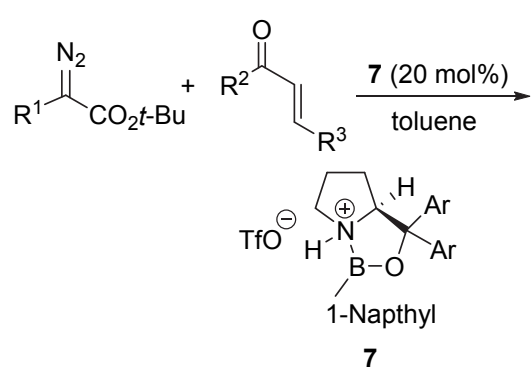<smiles>[R]C(=O)C1=NNC([R1])(OC(C)(C)C)C1[R]</smiles>

图式 18 手性噁唑硼烷催化的不对称 1,3-偶极子环加成反应 Scheme 18 Oxazaborolidinium ion (6/7) catalyzed enantioselective 1,3-dipolar cycloaddition
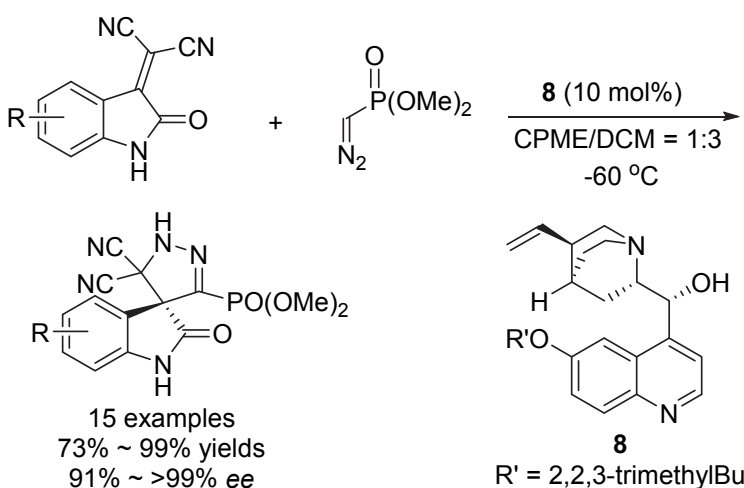<smiles>[R]Oc1ccc2nccc([C@@H](O)[C@@H]3C[C@H](C=C)C(C=C)=CN3)c2c1</smiles>

$\mathrm{R}^{\prime}=2,2,3$-trimethylBu

CPME = cyclopentyl methyl ether.

图式 19 金鸡纳碱催化的重氮磷酸酯的不对称环加成反应

Scheme 19 Cinchona alkaloids catalyzed asymmetric 1,3-dipolar cycloaddition of Seyferth-Gilbert reagent with isatylidene malononitriles

进一步地开发. 该方法可以从简单易得的原料直接出 发，通过串联脱乙酰基、脱水、或者分子内的 Michael 加成以及其他的分子内反应，来直接构筑高度官能化的 吡唑啉或者吡唑环体系. 其中, Smietana 等 ${ }^{[29]}$ 发展了以 Bestmann-Ohira 试剂为底物的三组分反应，合成了一系 列官能化的吡唑磷酸酯衍生物(Scheme 20). 首先, 醛、 腈衍生物和重氮磷酸酯在碱性条件下的三组分反应被 报道，反应经由 Knoevenagel 缩合、1,3-偶极子环加成和 后续的消除反应，区域选择性地构筑了吡唑环骨架 ${ }^{[29 a]}$. 进一步的，通过醛、甲基酮和 Bestmann-Ohira 试剂的 Claisen-Schmidt 反应、环加成和氧化串联反应，多取代 的 3-羰基-5-磷酰基吡唑衍生物被高效而直接地合成 ${ }^{[29 b]}$.

\section{2 与炔烃的环加成反应}

分子间的重氮化合物和炔烃，特别是贫电子炔烃的 


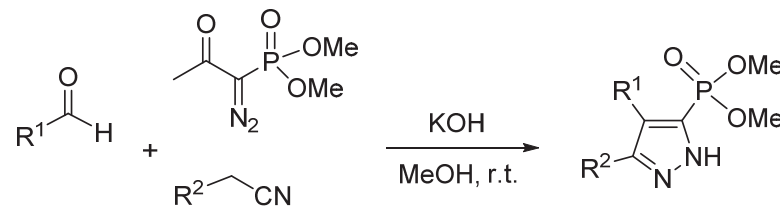

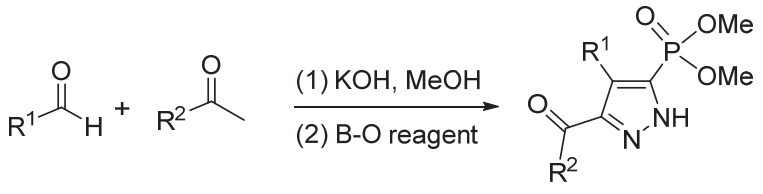

图式 20 三组分串联反应合成吡唑磷酸酯衍生物

Scheme 20 Three-component synthesis of phosphonyl pyrazoles from BOR

1,3-偶极子环加成反应，提供了一种重要的直接构筑吡 唑环的策略. 该转化具有良好的底物普适性, 炔烃组分 涵盖了端炔，环状炔烃，以及炔酮衍生物 ${ }^{[30]}$.

除了炔烃组分具有良好的官能团容忍性之外，重氮 底物的普适性也被人们进一步开发. 马军安课题组 ${ }^{[31]}$ 开发了银参与的原位产生的三氟甲基重氮甲烷和末端 炔烃的环加成反应(Scheme 21). 该反应可以在温和的 条件下，选择性地制备三氟甲基取代的吡唑衍生物. 该 类产物可以作为药物分子的核心骨架结构.

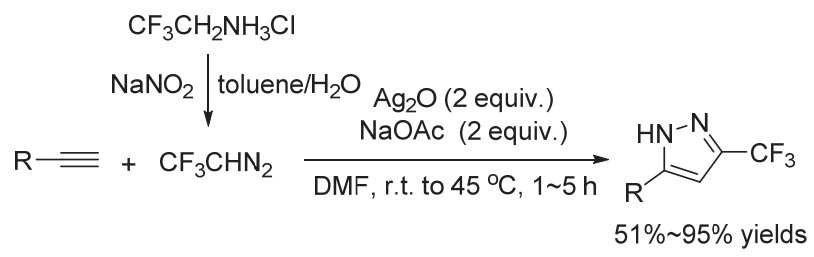

图式 21 银参与的端炔与 $\mathrm{CF}_{3} \mathrm{CHN}_{2}$ 的 1,3-偶极子环加成反应

Scheme $21 \mathrm{Ag}(\mathrm{I})$-mediated [3+2] cycloaddition of terminal alkynes with in situ generated $\mathrm{CF}_{3} \mathrm{CHN}_{2}$

Mykhailiuk 等 ${ }^{[32]}$ 进一步地将炔烃的 1,3-偶极子环加 成反应策略, 应用到其他与重氮中间体的反应中去. 他 们实现了原位生成的二氟甲基重氮甲烷 $\mathrm{CF}_{2} \mathrm{HCHN}_{2}$ 、以 及氰基重氮甲烷 $\mathrm{NCCHN}_{2}$ 与多种类型的炔烃以及烯烃 的环加成反应, 高效且选择性地制备了二氟甲基和氧基 取代的吡唑环(Scheme 22). 这些反应具有官能团容忍 性良好、反应条件温和等优点.

除此之外, 邻三甲基硅基苯酚三氟甲磺酸酯可以在 碱性或者氟负离子存在的条件下, 作为苯炔中间体的前 体, 和重氮化合物发生环加成反应, 直接制备在药物合 成中非常重要的取代的吲唑骨架 ${ }^{[33 a]}$. Yamamoto 课题 组 ${ }^{[33 b]}$ 通过选择 $\mathrm{CsF}$ 或者 $\mathrm{KF}$ 作为碱, 以及调节苯炔前体 和重氮烷衍生物的比例, 可以选择性地合成 $1 H$-吲唑或 者 $N$-芳基吲唑(Scheme 23).

Larock 等 ${ }^{[33 c]}$ 报道了底物控制的吲唑衍生物的多样 性合成途径. 当采用单取代的重氮烷作为底物时, 根据 反应物的投料比的差异, 可以选择性地控制制备 $1 H$-吲 唑或者 $N$-芳基吲唑; 当采用双羰基重氮作为底物时，可 以得到单一的 $N$-酰基吲唑衍生物(Scheme 24).
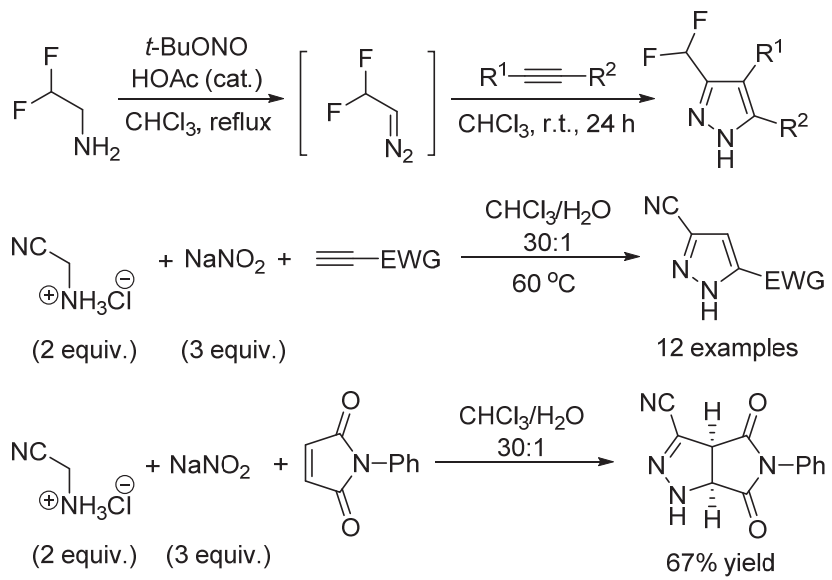

图式 $22 \mathrm{CF}_{2} \mathrm{HCHN}_{2}$ 与 $\mathrm{NCCHN}_{2}$ 与炔烃的 1,3-偶极子环加成反应 Scheme 22 1,3-Dipolar cycloaddition of in situ generated $\mathrm{CF}_{2} \mathrm{HCHN}_{2}$ and $\mathrm{NCCHN}_{2}$

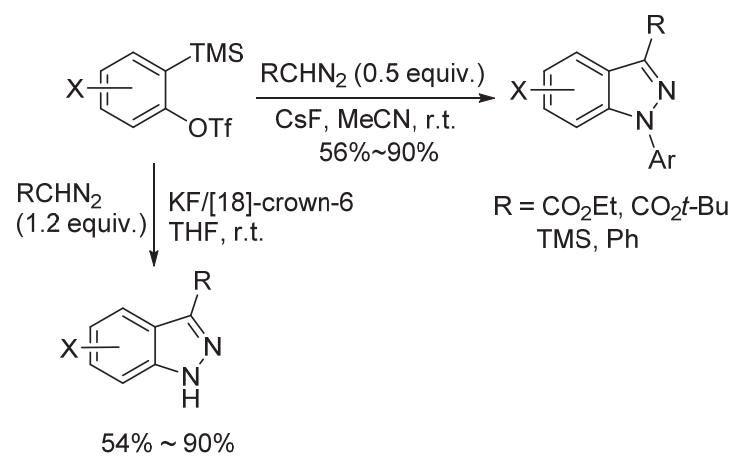

图式 23 重氮烷和苯炔中间体的环加成反应

Scheme 23 Cycloaddition of various diazomethane derivatives with arynes

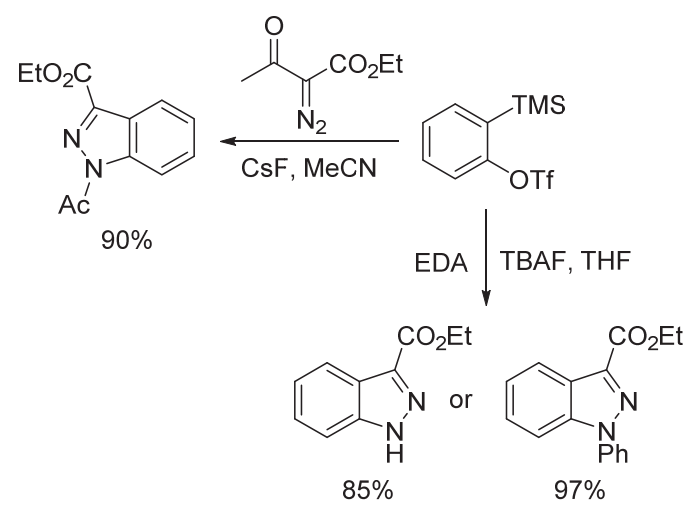

图式 24 底物控制的苯炔中间体和重氮化合物的环加成

Scheme 24 Substrate-dependent cycloaddition of arynes with diazo compounds

此外，银催化的苯炔中间体和羰基重氮化合物的环 加成反应被开发，用于选择性地合成 2 -芳基- $2 H$-吲 唑 ${ }^{[33 e]}$. 反应机理被认为是首先经过 1,3 -偶极子环加成 反应，然后经由去质子得到银物种 $\mathbf{9}$, 最后通过对苯炔 的亲核进攻得到目标杂环化合物(Scheme 25). 


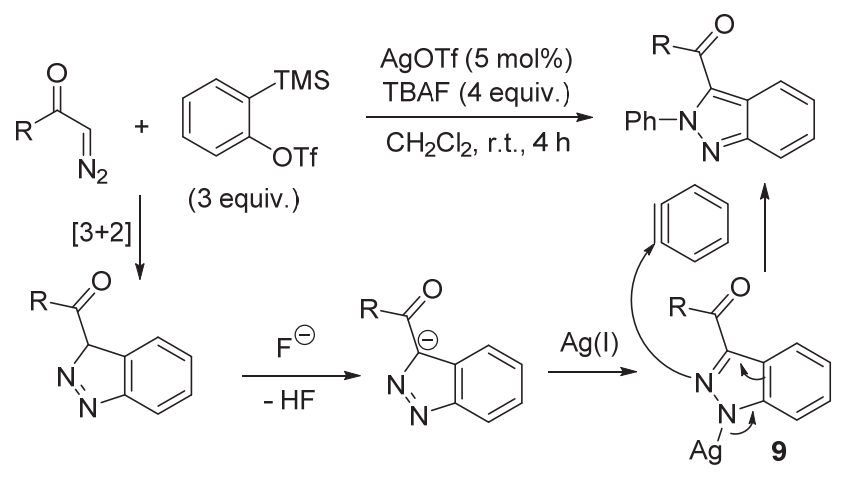

图式 252 -芳基- $2 H$-吲唑的合成

Scheme 25 Synthesis of 2-aryl-2H-indazoles

不仅重氮化合物可以发生该类型的环加成反应, 腙 类衍生物同样可以在碱性条件下发生反应来制备吲唑 和吡唑衍生物. 例如, 对甲苯磺酰腙可以在碱性条件下 作为重氮的前体, 和末端炔烃发生区域选择性的 1,3-偶 极子环加成反应. 该反应底物普适性较广, 涵盖了链状 或者环状羰基化合物衍生的腙, 以及具有光活性的腙衍 生物. 该策略可以制备不同类型的吡唑环体系，包括多 取代的、并环或螺环体系、以及含有手性中心的吡唑环 (Scheme 26) ${ }^{[34]}$.<smiles></smiles>

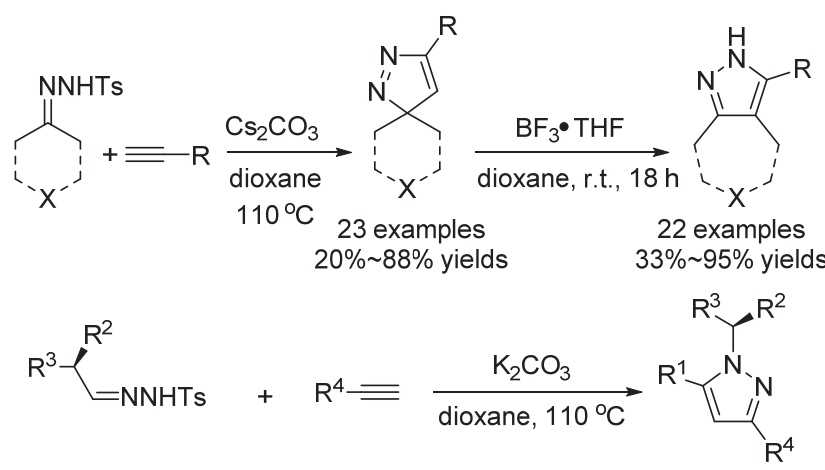

图式 26 对甲苯磺酰腙和炔烃的区域选择性环加成反应

Scheme 26 Regioselective cycloaddition of tosylhydrazones with alkynes

\section{3 与联烯的环加成反应}

重氮和联烯的 1,3-偶极子环加成反应既可以在分子 内, 也可以在分子间进行. 对于分子内的过程, 一个典 型的例子, 是从非环状的底物来直接构筑并环体系 triquinanes (Scheme 27) ${ }^{[35]}$. 该反应经历了重氮中间体和 联烯的选择性环加成、高温下放出氮气得到双自由基 10、以及后续的自由基 $[3+2]$ 环加成过程. 该策略从较 为简单易得的重氮前体出发, 通过无金属参与的一步串 联反应, 经由连续的环加成反应, 为制备官能化的五并
五并五体系提供了一条直接而简便的途径.

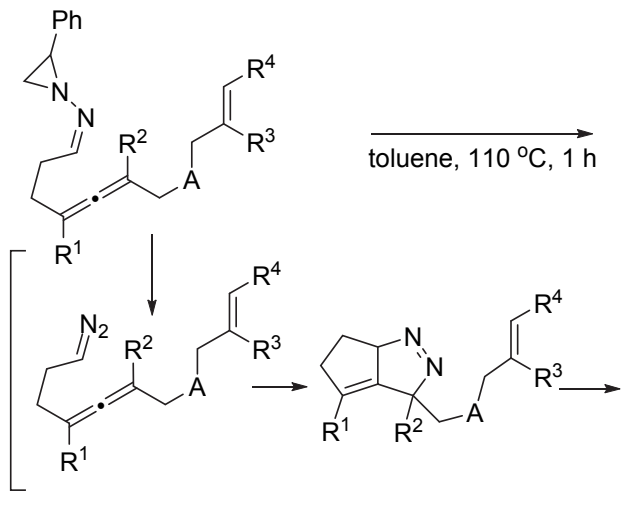<smiles>[R]C1=C2C([R])C3CCC(CC3)C2([R])CC1</smiles><smiles>[R]C/C=C\CC[B]C(=C)CC(=O)C([R1])[Y]</smiles>

图式 27 串联的重氮和联烯的环加成反应合成 triquinanes 衍生物 Scheme 27 Tandem cycloaddition strategy for synthesizing fused triquinanes

此外, 马军安课题组 ${ }^{[36]}$ 报道了原位产生的三氟甲 基重氮甲烷和贫电子的联烯化合物发生选择性环加成 的过程. 该反应可以在温和的条件下，通过对于溶剂和 碱的调控，可以选择性地制备三氟甲基取代的吡唑啉或 者吡唑衍生物(Scheme 28).

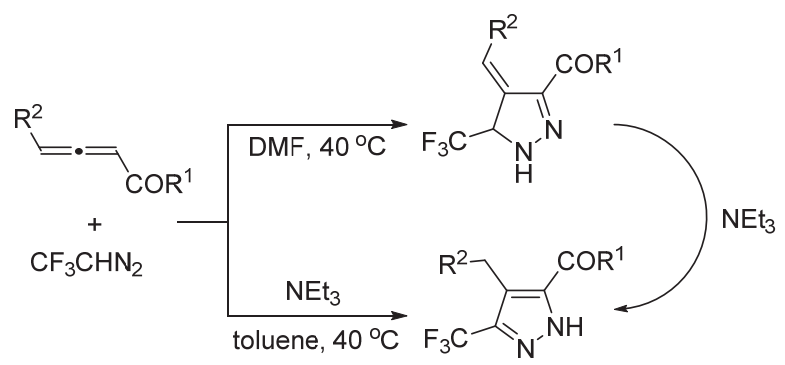

图式 28 重氮和联烯的环加成制备三氟甲基取代的吡唑啉衍生物 Scheme 28 Synthesis of $\mathrm{CF}_{3}$-substituted pyrazolines and pyrazoles by cycloaddition of allenes with $\mathrm{CF}_{3} \mathrm{CHN}_{2}$

\section{4 与其它亲偶极子的环加成反应}

除了烯烃、炔烃以及联烯之外，更多的环加成反应 组分也被人们开发, 包括了硫代羰基化合物 ${ }^{[37]}$ 、单线态

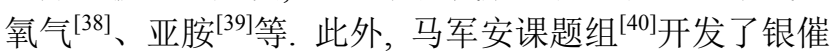
化的三氟甲基重氮甲烷和芳基重氮盐中间体的 1,3-偶极 子环加成反应. 该反应在较低的温度下进行，从廉价易 得的原料出发, 可以选择性地制备三氟甲基衍生的四唑 类骨架(Scheme 29). 从商业可得的芳香胺出发的一锅 法步骤也进一步地被实现.

此外, 马军安课题组 ${ }^{[41]}$ 还开发了银催化的重氮甲 烷衍生物和异腈的 1,3-偶极子环加成反应(Scheme 30). 该反应具有条件温和, 选择性好, 底物普适性广泛的特 


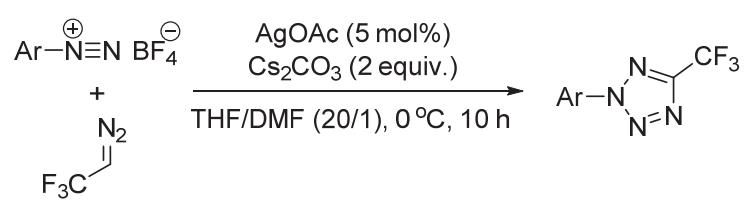

(2 equiv.)

图式 29 银催化的三氟甲基重氮甲烷和芳基重氮盐的环加成 Scheme 29 Ag-catalyzed [3+2] cycloaddition of aryldiazonium salts with $\mathrm{CF}_{3} \mathrm{CHN}_{2}$

点, 重氮组分涵盖了三氟甲基重氮甲烷, 重氮酯以及三 甲基硅基重氮甲烷等. 在银催化剂和温和的反应条件 下，一系列三氟甲基或其它取代基衍生的三氮唑骨架被 直接而高效地组装. 反应的机理涉及到异腈的银物种和 重氮的环加成, 以及银卡宾中间体 $\mathbf{1 1}$ 的产生. 除了异腈 之外, 腈类化合物目前也被开发作为环加成反应组分, 用以高效而选择性地合成取代的三氮唑骨架 ${ }^{[42]}$.

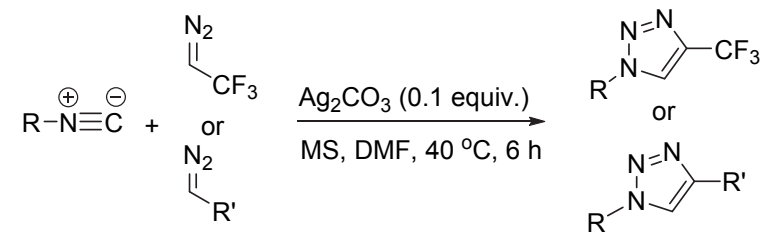

mechanism

$$
\begin{aligned}
& \text { mechanism }
\end{aligned}
$$

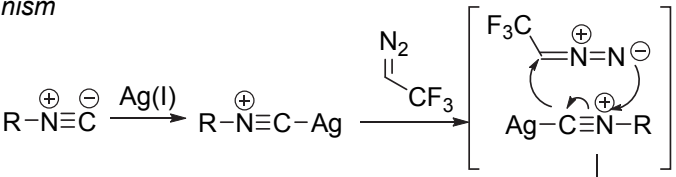

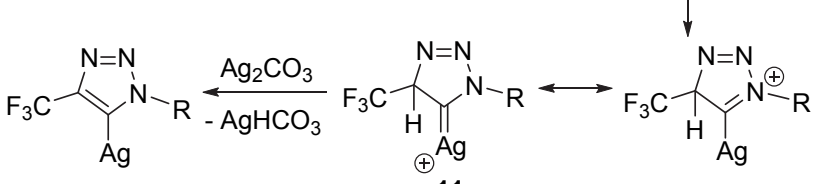

$$
\begin{aligned}
& \mathrm{AgHCO}_{3} \downarrow-\mathrm{Ag}_{2} \mathrm{CO}_{3} \\
& \stackrel{\mathrm{N}=\mathrm{N}}{\mathrm{N}} \underset{\mathrm{R}}{\mathrm{N}} \backslash \mathrm{CF}_{3}
\end{aligned}
$$

图式 30 银催化的重氮化合物和异腈的 1,3-偶极子环加成

Scheme $30 \mathrm{Ag}(\mathrm{I})$-catalyzed 1,3-dipolar cycloaddition of diazo compounds with isocyanides

\section{4 重氮化合物作为亲电试剂的反应}

重氮化合物的氮原子可以作为亲电试剂来接受电 子或者亲核试剂的进攻; 通过这种反应途径, 可以合成 多种类型的目标分子或者芳杂环. 其中, 有机磷试剂参 与的重氮化合物的 Diaza-Wittig 反应提供了一种传统、 直接而有效的构筑哒嗪衍生物的方法. 反应的途径经由 磷原子对于重氮的端位氮原子的亲核进攻生成磷杂二 嗪 12, 然后发生分子内的 Wittig 反应来构筑目标哒嗪环 (Scheme 31). 分子内的 Diaza-Wittig 反应具有良好的官 能团容忍性和底物普适性, 已经被用来合成多样化取代 的氮杂芳香环 ${ }^{[43]}$.<smiles>[R]C(=O)CC([R])=O</smiles>

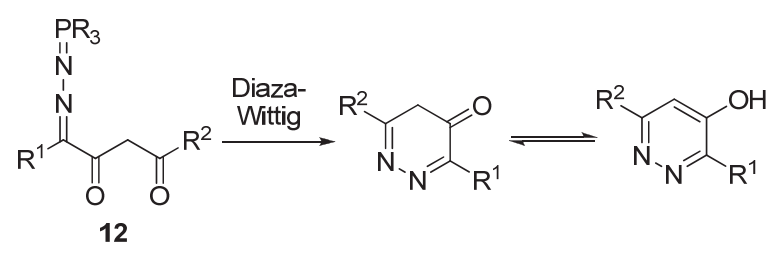

图式 31 分子内 Diaza-Wittig 反应的一般途径

Scheme 31 General pattern of intramolecular Diaza-Wittig reaction

此外，有机小分子催化的一锅法制备多取代的哒嗪 骨架也被开发 ${ }^{[44]}$. 该反应以重氮烷烃衍生物和 MoritaBaylis-Hillman 碳酸酯为原料, 经由碱促进的亲核取代 反应、以及后续的 Diaza-Wittig 反应来直接构筑四取代 的哒嗪衍生物(Scheme 32).

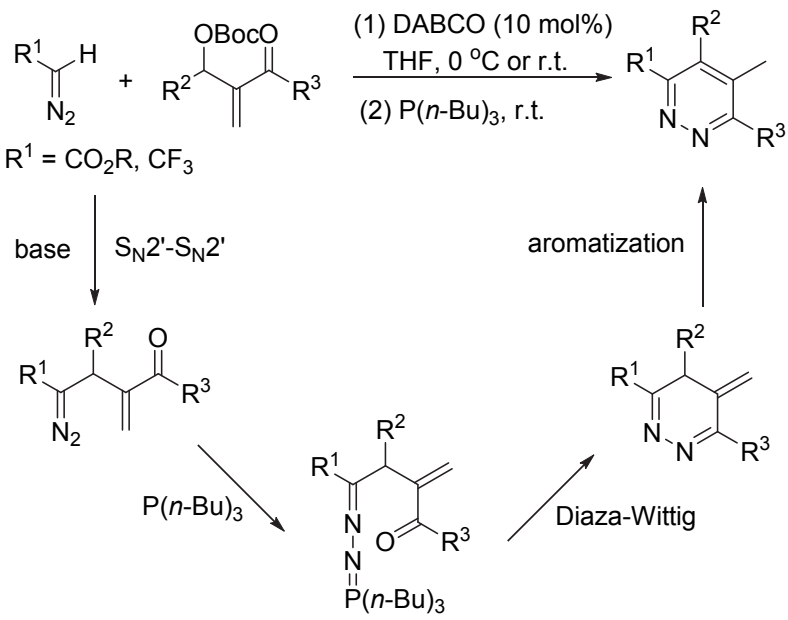

图式 32 从重氮和 MBH 碳酸酯出发的四取代哒嗪衍生物的一锅法合 成

Scheme 32 One-pot synthesis of pyridazines from MBH carbonates with diazo compounds

除了磷试剂之外, 其它的亲核试剂进攻重氮组分端 位的氮原子的反应也被报道. 例如，腙衍生物不仅可以 通过磷试剂和重氮羰基化合物的反应来制备，也可以通 过格氏试剂或者锂试剂对重氮的进攻来获得. 制备的腙 中间体可以通过进一步的转化，来合成多取代的吡咯骨 架 $(\text { Scheme 33) })^{[45]}$.

除了吡咯骨架之外, 三唑类衍生物亦可以通过重氮 化合物在无过渡金属参与的条件下直接进行制备. 在碱 的作用下, $\alpha, \alpha$-二氯对甲苯磺酰腙可以和一级胺发生分 子间的取代，进一步发生分子内的氮原子对于重氮的亲 核进攻，从而得到 1,4-二取代的三氮唑 ${ }^{[46 a]}$. 另外一例制 备三氮唑啉的报道涉及到了碱促进的 $\beta$-氨基- $\alpha$-重氮酯 和卤化物的反应，能够以很高的非对映选择性来获得高 度官能化的三氮杂五元环骨架 ${ }^{[46 b]}$ (Scheme 34). 


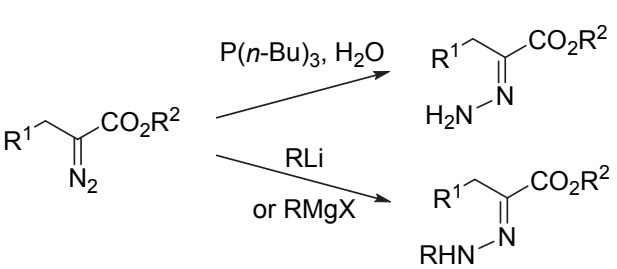

$$
\begin{aligned}
& \longrightarrow \mathrm{R}^{2} \mathrm{O}_{2} \mathrm{C}
\end{aligned}
$$

图式 33 从 $\alpha$-重氮酯来制备腙类化合物

Scheme 33 Synthesis of hydrazones from corresponding $\alpha$-diazo esters

$$
\underset{\mathrm{R}^{2} \mathrm{NH}_{2}}{\stackrel{(i-\mathrm{Pr})_{2} \mathrm{NEt}}{\mathrm{MeCN} / \mathrm{EtOH}, \text { r.t. }}}[\underbrace{\mathrm{NNHTs}}_{\mathrm{NHR}^{2}}[\underbrace{\mathrm{Cl}_{2}}_{\mathrm{R}^{1}}] \longrightarrow
$$

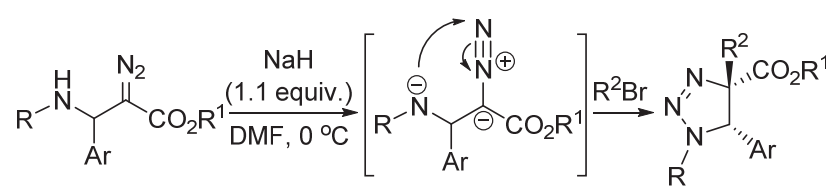

图式 34 无过渡金属参与的分子内亲核取代反应制备三氮唑衍生物 Scheme 34 Transition-metal-free synthesis of triazoles and triazolines via intramolecular nucleophilic addition

最近, 刘群和赵玉龙课题组 ${ }^{[47]}$ 开发了 DBU 介导的 重氮羰基化合物和活泼亚甲基化合物的偶联反应，实现 了 $\mathrm{C}-\mathrm{N}$ 键的选择性构筑. 该反应提供了一种合成肼类 衍生物的温和而高效的方法, 并且, 目标产物可以经由 进一步的转化来制备含氮杂环. 从反应的机理角度上 看, DBU 既活化了重氮组分, 也通过去质子的过程活化 了亚甲基化合物而得到烯醇式中间体 13 , 促进了后续 的 $\mathrm{C}-\mathrm{N}$ 键构筑过程生成 14, 最后经由 DBU 参与的互 变异构得到最终产物(Scheme 35).

2011 年, 冯小明课题组 ${ }^{[48]}$ 开发了一种不对称的高 效构筑 C- $\mathrm{N}$ 键的方法(Scheme 36). 该反应从取代的重 氮酯和廉价易得的酮出发, 在 Lewis 酸、碱的存在时, 以 及双氮氧酰胺 15 作为手性助剂的条件下, 实现了酮羰 基 $\alpha$ 位的高度区域选择性和立体专一性的 $\mathrm{C}-\mathrm{N}$ 键构筑. 该反应具有良好的产率(高达 $98 \%$ ) 和非常好的对映选择 性(高达 $99 \%$ 的 ee 值).

Gois 和合作者 ${ }^{[49]}$ 开发了碱性条件下氮杂环卡宾催 化的醛和重氮化合物的 $\mathrm{C}-\mathrm{N}$ 键构筑反应(Scheme 37). 该反应可以作为合成 $N$-酰基腙类衍生物的补充方法. 特别地, 对于呋喃醛的衍生物, 可以高效而直接的合成 5-羊货甲基呋喃衍生物, 该类产物具有一定的生物活性. 反应机理首先涉及到 Breslow 中间体 $\mathbf{1 6}$ 的生成, 进而发 生对重氮组分的亲核进攻而完成反应.

在手性的咪唑啉衍生的膦配体 17 的存在下, 钯催 化的以烯基酯基取代的环丙烷和 3-重氮氧化吲哚为原

$$
\widehat{R}_{E W G}^{+} \mathrm{R}^{1} \prod_{\mathrm{O}}^{\mathrm{N}_{2}} \mathrm{R}^{2} \frac{\mathrm{DBU}(0.5 \text { equiv. })}{\mathrm{DMF} \text {, r.t., } 1 \sim 30 \mathrm{~min}}
$$

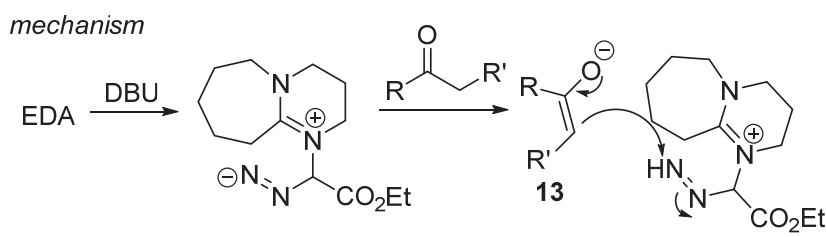

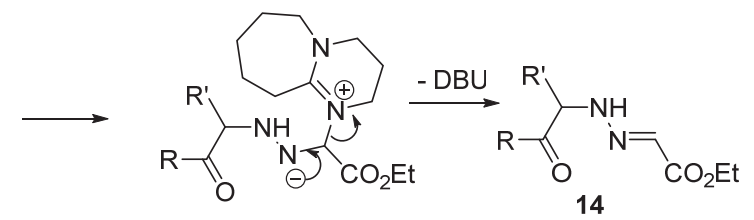<smiles>[R]C(=O)/C([R])=N/NCCOCC</smiles>

图式 $35 \mathrm{DBU}$ 介导的重氮羰基化合物与活泼亚甲基化合物的偶联 Scheme 35 DBU-mediated coupling reaction of $\alpha$-diazocarbonyls as $N$-terminal electrophiles

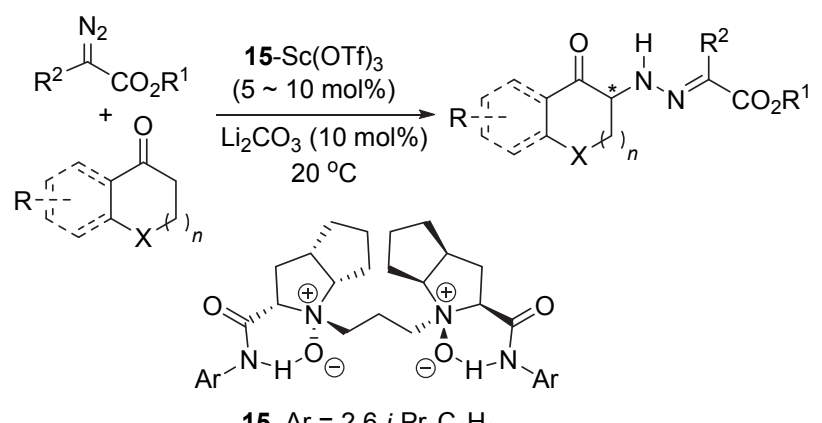

15, $\mathrm{Ar}=2,6-i-\mathrm{Pr}_{2} \mathrm{C}_{6} \mathrm{H}_{3}$

图式 36 重氮酯和酮衍生物的不对称 C- $\mathrm{N}$ 键构筑反应 Scheme 36 Enantioselective $\mathrm{C}-\mathrm{N}$ bond formation of ketones with $\alpha$-diazoesters

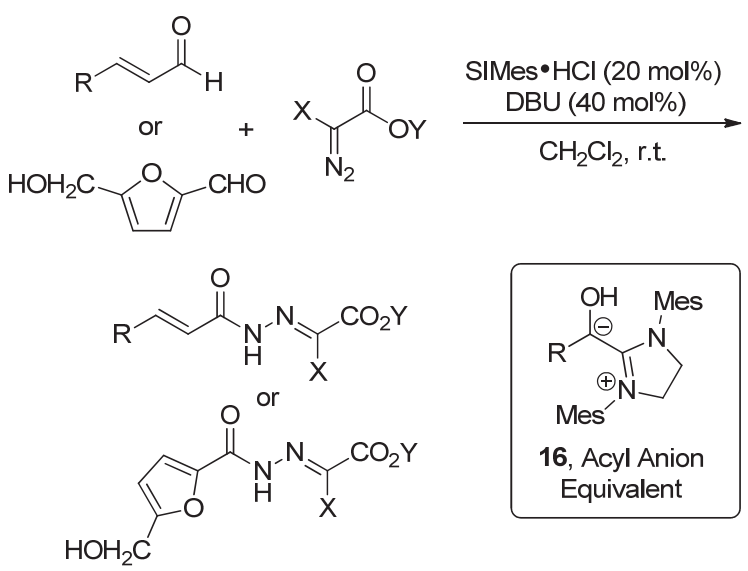

图式 $37 \mathrm{NHC}$ 催化的醛和重氮酯的反应构筑 $N$-酰基腙

Scheme 37 NHC-catalyzed synthesis of $N$-acylhydrazones from aldehydes with diazoesters

料的不对称 $[3+2]$ 环加成反应被施敏课题组 ${ }^{[50]}$ 报道，提 供了一种新颖而原子经济性的合成吡唑啉的方法 
(Scheme 38). 进一步地, 该目标分子可以和马来酰亚胺 发生后续的环加成反应, 高度立体专一性地制备含有多 个手性中心的螺环吡唑啉的骨架. 该策略实现了多手性 中心的复杂杂环骨架的直接而高效的构筑过程.
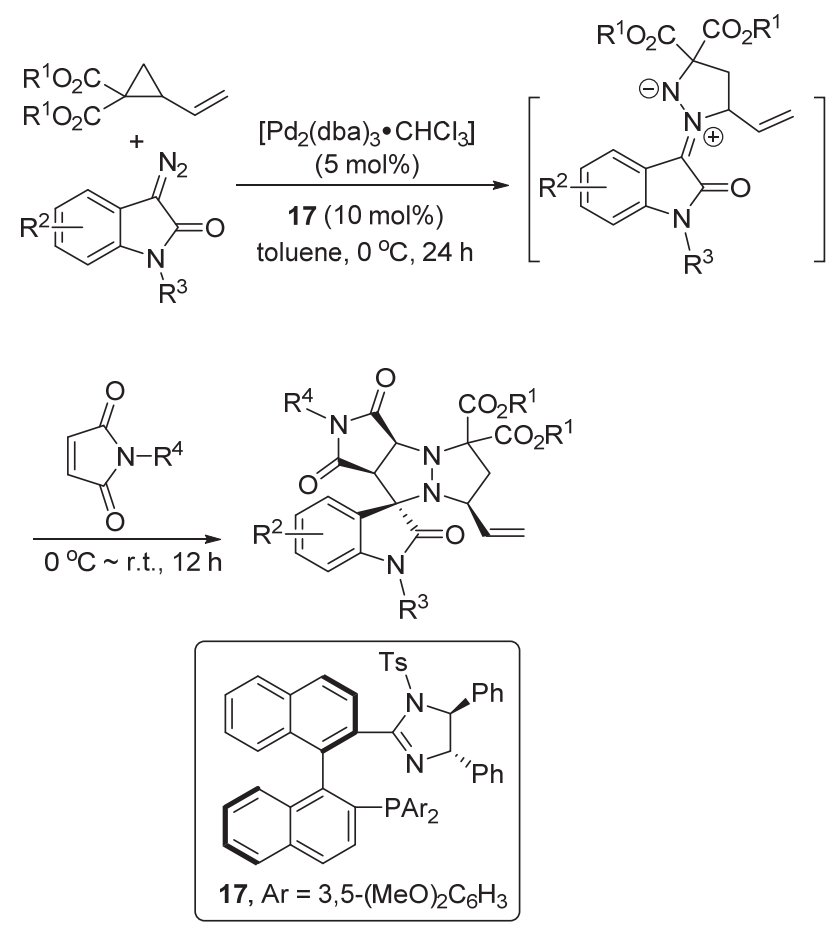

图式 38 钯催化不对称的氧化吲哚衍生的螺环吡唑烷串联合成

Scheme 38 Pd-catalyzed asymmetric tandem synthesis of oxindole-fused spiropyrazolidines

最近，一例三价铁催化的重氮化合物和烯烃的偶联 反应被报道, 通过 $\mathrm{C}-\mathrm{N}$ 键区域选择性的构筑, 实现了 形式上的烯烃的氢胺化反应(Scheme 39) ${ }^{[51]}$. 反应的机 理被认为经过了自由基的过程, 涉及到二价铁和三价铁 的循环、以及烷基自由基对于重氮化合物的加成，从而 制备了腙的衍生物.

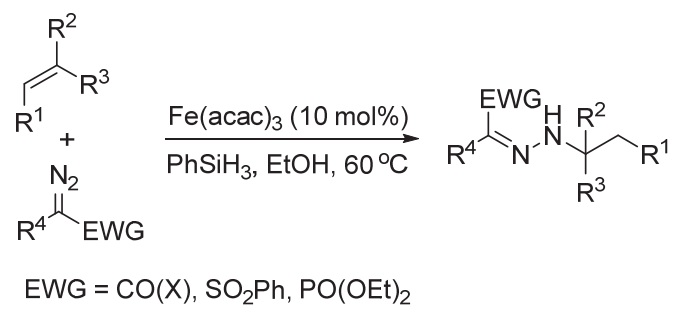

图式 39 铁催化的重氮化合物与烯烃的自由基类型偶联反应

Scheme 39 Fe-catalyzed radical-type olefin hydroamination with diazo compounds

\section{5 烯基重氮化合物的分子内反应}

烯基重氮羰基化合物或者烯基重氮中间体的分子 内环化反应可以直接构筑取代的吡唑衍生物. 典型的分 子内环化反应途径一般经由 1,5 -电环化, 然后通过 1,5迁移来获得取代的吡唑环 (Scheme 40). 一方面, 该途径
通常作为烯基重氮化合物参与转化的副反应过程而存 在; 另一方面，目前为止，该方法已经作为一种成熟的 合成氮杂环的步骤，人们更多的去关注底物的拓展和官 能团的容忍性、取代基的影响，以及开发从廉价易得的 原料出发的一锅法的制备步骤等.

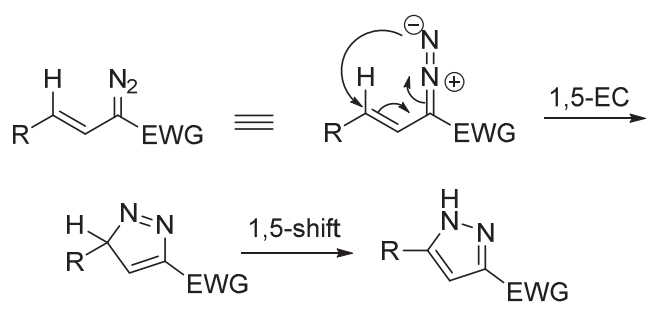

图式 40 烯基重氮经由 1,5-电环化过程构筑吡唑环机理示意图 Scheme 40 Reaction mechanism for 1,5-electrocyclization of vinyl diazo compounds

代表性的一例工作，是 2011 年 Frantz 等 ${ }^{[52]}$ 报道的 将交叉偶联反应与电环化策略相结合的合成方法. 该反 应以双羰基化合物的烯醇三氟甲磺酸酯和重氮化合物 为原料, 在钯催化剂的条件下，直接合成三取代的吡唑 环(Scheme 41). 反应途径经由钯催化的重氮酯与烯醇 酯的交叉偶联原位产生烯基重氮中间体 18, 进而在一 定温度下发生分子内关环得到目标杂环。
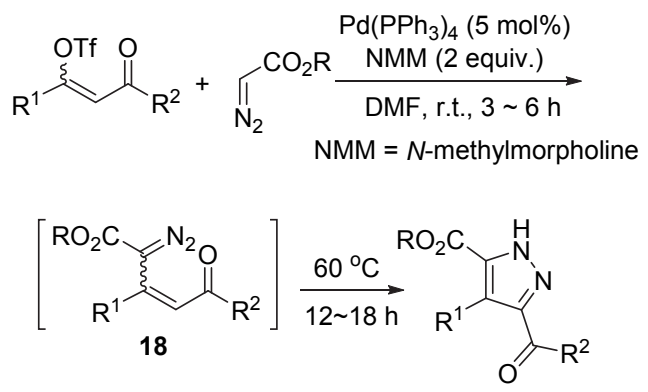

图式 41 钯催化交叉偶联与分子内关环策略相结合的实例 Scheme 41 Pd-catalyzed coupling/electrocyclization process

此外, 相同的课题组进一步开发了一种电环化反应 与芳香环 Cope 重排结合的策略, 以烯基重氮为原料, 在较为温和的条件下, 得到了重排的吡唑环衍生物 (Scheme 42) ${ }^{[53]}$.

一例钴催化的芳基重氮盐和烯基重氮化合物特殊 的偶联反应被孙江涛课题组 ${ }^{[54]}$ 开发，用于合成 $N$-芳基 化的吡唑环(Scheme 43). 该反应首先经由氧化加成得 到芳基钴物种 19, 然后和重氮的氮原子配位，进一步促 进了 1,5-电环化反应; 然后通过质子迁移和 20 的还原消 除过程得到了吡唑环产物. 该反应不仅提供了一种简便 的官能化的吡坐的选择性制备方法，而且开创了芳基重 氮盐偶联和电环化反应的组合策略.

2015 年, 孙江涛课题组 ${ }^{[55]}$ 进一步研究了金催化的 烯基重氮化合物和芳基酯基重氮的选择性交叉偶联反 应，区域和化学选择地制备了 $N$-官能化的吡唑衍生物 


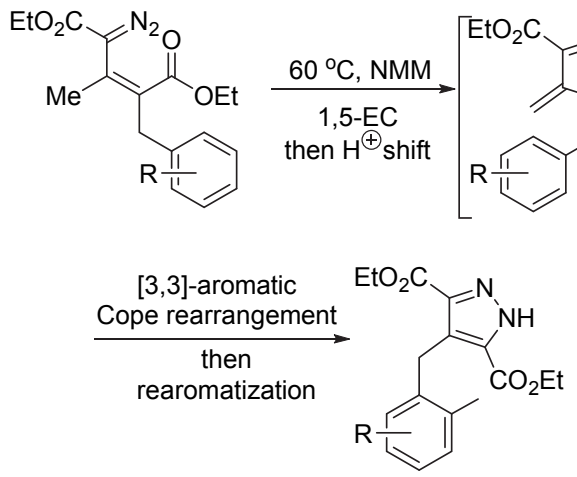

图式 42 分子内电环化与芳香环 Cope 重排的串联反应

Scheme 42 Intramolecular electrocyclization process and aromatic Cope rearrangement

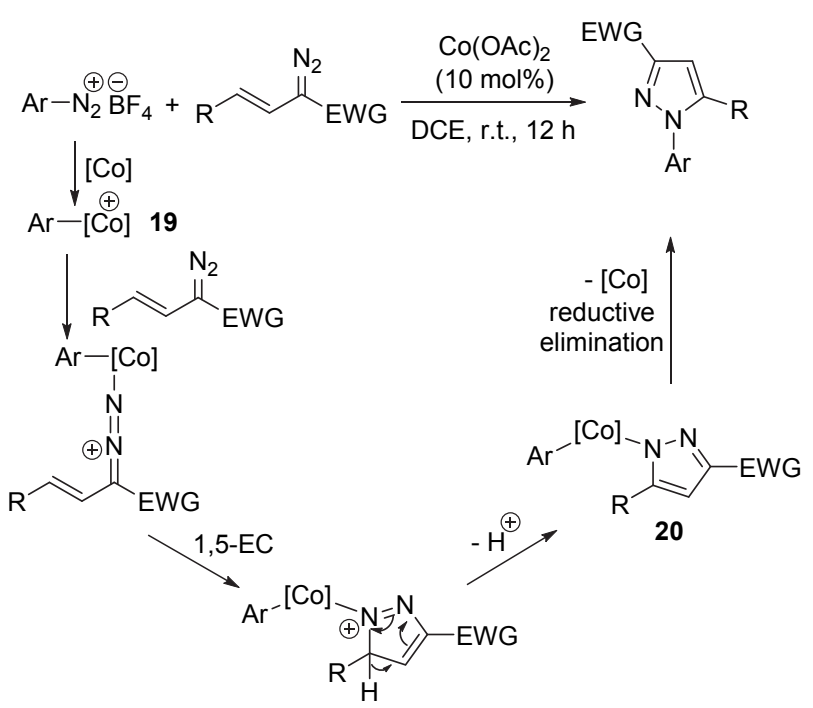

图式 43 钴催化的芳基重氮盐和烯基重氮的交叉偶联反应

Scheme 43 Co-catalyzed cross-coupling reaction of aryldiazonium salts with vinyl diazoacetates

(Scheme 44). 该反应的选择性是由金催化剂的配体调 控的，利用了配体和芳基酯基重氮组分的空间位阻而实 现. 反应机理涉及到金卡宾 21 或 22 的选择性生成, 然 后经由另一分子重氮组分氮原子的亲核进攻, 以及后续 的环化过程, 完成了吡坐环的构筑.

除了吡唑环之外，1,2,3-三氮唑体系也可以通过采 用亚胺基重氮为原料或者原位产生的亚胺基重氮中间 体, 经由 1,5 -电环化过程来完成构筑 ${ }^{[56-58]}$. 因此, 该类 烯基重氮衍生物的分子内电环化反应是一种很好的构 筑多氮原子杂环体系的直接而简便的方法.

此外, 2015 年, 邓桂胜课题组 ${ }^{[59]}$ 报道了经由分子内 的电环化反应及串联过程, 构筑了更为复杂的吡喃酮并 吡唑体系. 该反应从重氮衍生的炔基酮出发, 在有机碱 存在的温和条件下, 经过无金属参与的分子内 $6 \pi$ 电子 环化, 以及后续的分子内 Michael 加成过程, 一步直接 构筑了羰基取代的吡喃酮并吡唑骨架(Scheme 45). 该 反应具有官能团容忍性良好、原子经济性高以及条件温 和等特点.
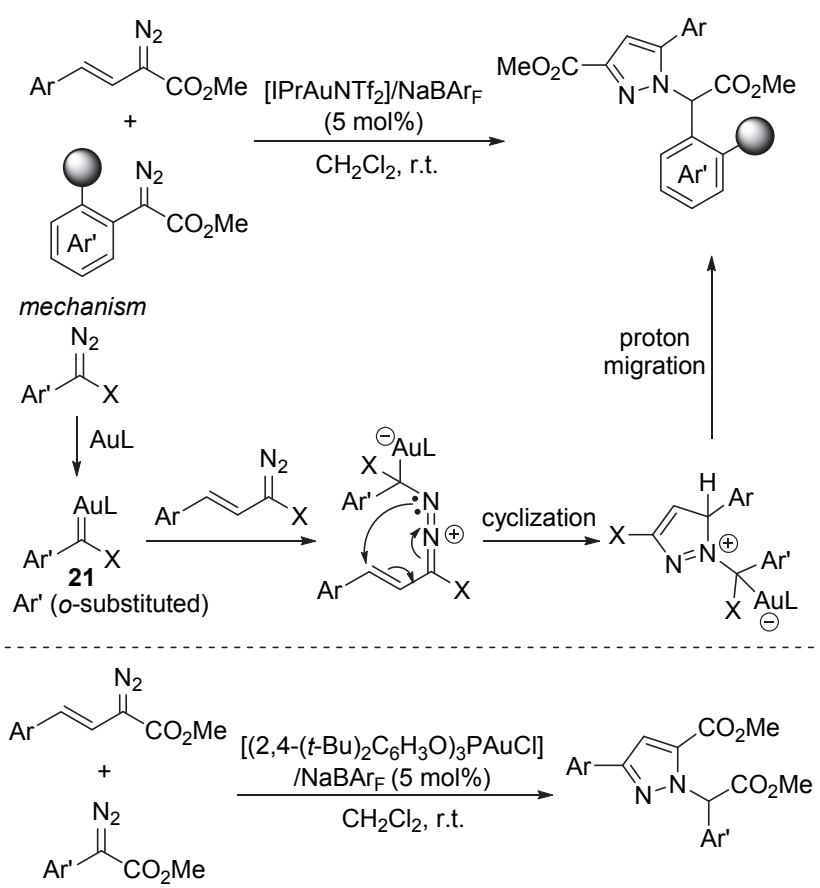

mechanism

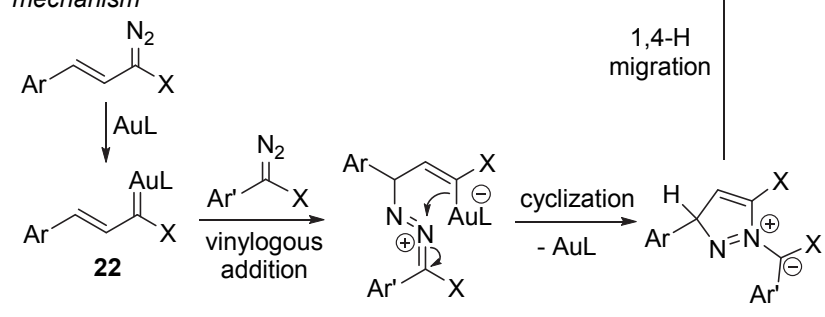

图式 44 金催化的烯基重氮和芳基酯基重氮的选择性交叉偶联反应 合成 $N$-取代的吡唑衍生物

Scheme $44 \mathrm{Au}(\mathrm{I})$-catalyzed selective cross-coupling of diazo compounds to afford $N$-substituted pyrazoles in position-switchable mode

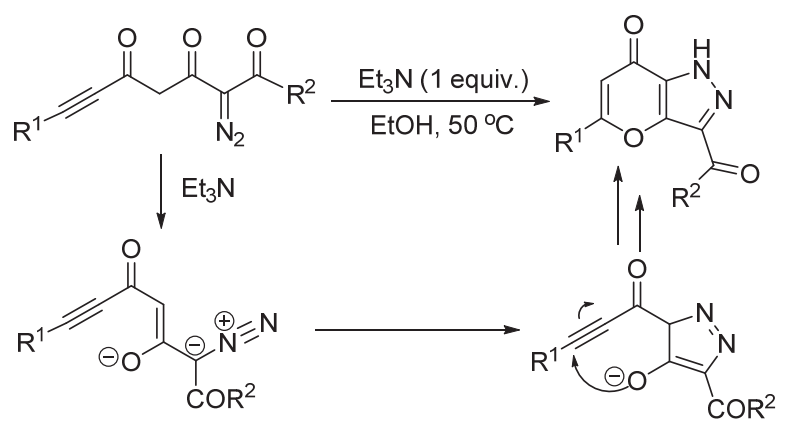

图式 45 从重氮衍生的炔基酮合成吡喃酮并吡唑体系

Scheme 45 Synthesis of pyrano[3,2-c]pyrazol-7(1H)-one from 2-diazo-3,5-dioxo-6-ynoates

\section{6 重氮化合物的还原反应}

重氮化合物可以和含有形式上的氢负离子的还原 剂反应，生成氮基团保留的还原产物. 此外, 在钯催化 氢化的条件下发生对甲苯磺酰腙的还原，可以得到羰基 化合物形式上脱氧氢化的还原产物，此时氮基团没有保 留，以氮气的形式离去 ${ }^{[60]}$. 因此，通过控制还原反应的 条件，可以选择性地得到氮基团保留的部分还原产物或 
者脱氮气的彻底还原产物.

2009 年，一例三步的 $\alpha$-氨基酸酯的脱氨基过程被 报道 ${ }^{[61]}$. 该步骤经历了氨基的重氮化、重氮化合物的还 原、以及 $\mathrm{DBU}$ 参与的还原反应并伴随着氮气的离去, 高 效地制备了一系列官能化的脱氨基羧酸酯产物. 其中, 在嗍氢化钠作为还原剂的条件下, $\alpha$-重氮酯被选择性地 还原到对应的腙 23 (Scheme 46).

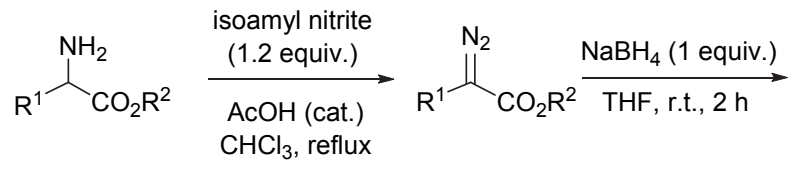

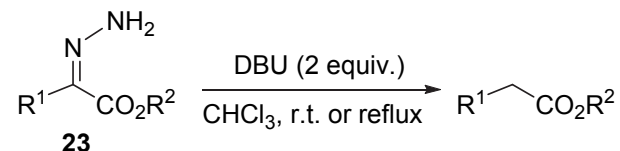

图式 46 经由重氮化合物还原的 $\alpha$-氨基酸酯的脱氨基过程

Scheme 46 Reduction sequence involving the deamination of $\alpha$-aminoesters

此外, Nishida 课题组 ${ }^{[62]}$ 报道了 3-卤代芳环-3-羟基2-重氮丙酸酯的还原反应. 该反应以三乙基硼氢化锂作 为还原剂, 高效地制备了对应的腙 24; 并且, 制备的腙 或者肼的衍生物可以进一步通过后续的转化, 得到官能 化的含氮杂环, 例如 1-氨基吲哚啉等(Scheme 47). 该类 还原反应及串联策略提供了一种简便而高效的制备含 有两个氮原子的环状目标分子骨架的方法.

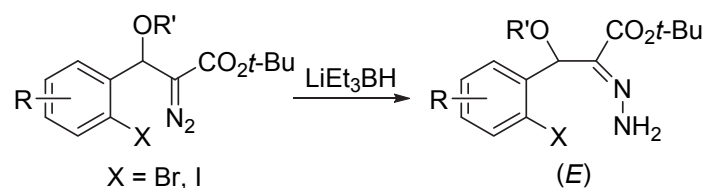
$\mathrm{X}=\mathrm{Br}, \mathrm{I}$

$(E)$

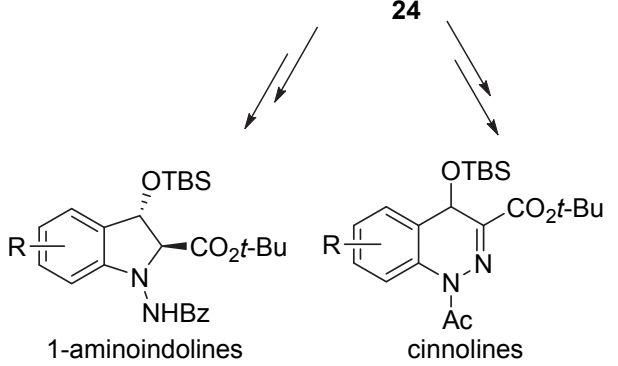

图式 47 经由 3-卤代芳环-3-羟基-2-重氮丙酸酯的还原反应制备腙以 及合成杂环的应用

Scheme 47 Preparation of hydrazones from corresponding 3-haloaryl3-hydroxy-2-diazopropanoates and applications in the synthesis of cinnolines and 1-aminoindolines

\section{7 重氮化合物的其它反应}

2011 年, 一例独特的铜催化的烯基重氮化合物和 高价碘试剂的氧化反应被 Barluenga 课题组 ${ }^{[63]}$ 报道, 可 以用来合成氧代重氮羰基化合物. 该选择性的氧化反应 过程经过了双键对于高价碘的进攻, 以及碘一氧杂四元 环的中间体, 最后伴随着碘苯的离去, 得到了 $\beta$ 位氧代
的重氮化合物(Scheme 48).

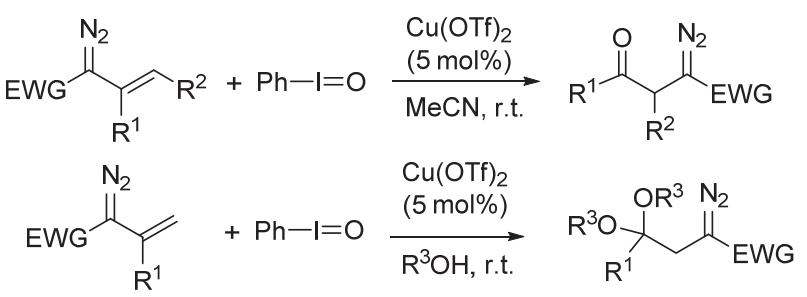

图式 48 铜催化的烯基重氮化合物和氧化碘苯的反应制备 $\beta$-氧代重 氮化合物

Scheme $48 \mathrm{Cu}$-catalyzed oxidative rearrangement to obtain $\beta$-oxodiazo compounds

此外, 一例特殊的铑催化的芳香环 $\mathrm{C}-\mathrm{H}$ 键官能团 化反应被孙江涛课题组 ${ }^{[64]}$ 开发. 该反应没有预先在芳 香环上引入导向基团，而是首先经由两分子芳基重氮化 合物的反应，得到二嗪类中间体 25 ; 进一步地，二嗪作 为导向基团，实现了芳香环选择性的 $\mathrm{C}-\mathrm{H}$ 键金属化， 发生后续的对三键的插入反应等(Scheme 49). 该反应 巧妙地利用了重氮化合物双分子的反应(通常作为副反 应的存在), 经过氮保留的偶联过程, 原位地引入了导 向基团, 然后通过铑催化的 $\mathrm{C}-\mathrm{H}$ 键活化过程完成转化, 合成了官能化的异喹啉骨架.

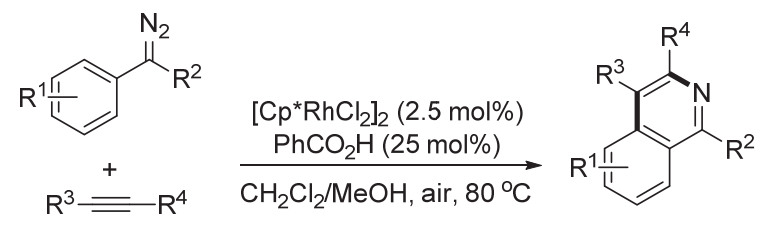

mechanism
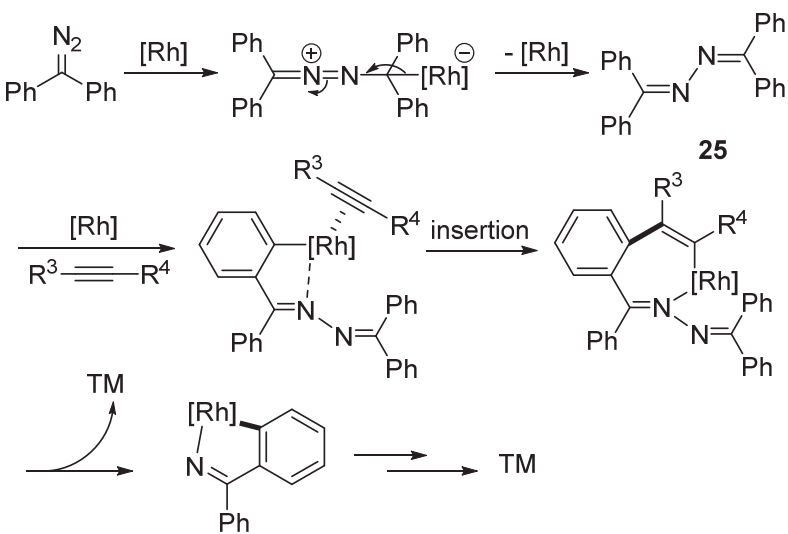

图式 49 铑催化的经由原位引入导向基团的芳基重氮化合物的 $\mathrm{C}-\mathrm{H}$ 官能团化

Scheme 49 Rh-catalyzed $\mathrm{C}-\mathrm{H}$ functionalization of aryl diazo compounds assisted by in situ generated directing group

\section{8 总结与展望}

重氮化合物或中间体以其多样化的反应性能，以及 较为简便的制备或产生方法, 引起了化学家们的极大研 究兴趣. 其中, 重氮化合物通过脱除氮气来作为卡宾或 者类卡宾前体的反应，已经得到了长足的发展和进步. 
相比较而言，重氮化合物的氮基团保留的反应，受关注 的程度则较低. 另一方面, 该类反应可以为目标功能分 子选择性地提供一个或者若干氮基团, 因此在合成含氮 的生物活性分子, 尤其含氮脂肪杂环或者芳杂环的骨架 合成中, 具有极其重要的作用. 本文总结了目前已有的 反应类型，根据反应的机理和途径进行了分类归纳.

在今后的研究中, 重氮化合物的氮基团保留的反应 面临的挑战包括以下几点: (1)进一步丰富和完善不对称 的 $\mathrm{C}-\mathrm{N}$ 键构筑. 一方面进一步拓展底物的普适性, 不 仅局限于羰基 $\alpha$ 位或者活泼亚甲基化合物，使其成为一 种普适性的 $\mathrm{C}-\mathrm{N}$ 键构筑过程; 另一方面需要提高反应 的区域选择性和立体选择性. (2)将氮基团保留的反应进 一步与过渡金属催化的交叉偶联反应、惰性 $\mathrm{C}-\mathrm{H}$ 键活 化过程、以及官能团转化反应相结合, 从而实现多种反 应策略的选择性调控, 拓展反应的类型. (3)发展自由基 类型的重氮化合物的氮基团保留的反应, 将自由基化学 与重氮化学进一步关联, 不仅具有理论上的意义, 而且 具有潜在的合成应用价值. (4)将该类反应, 尤其是重氮 化合物的 1,3-偶极子环加成反应等, 运用到天然产物、 药物和生命活性分子的全合成中, 提高这一系列反应的 实际应用价值.

\section{作者简介}

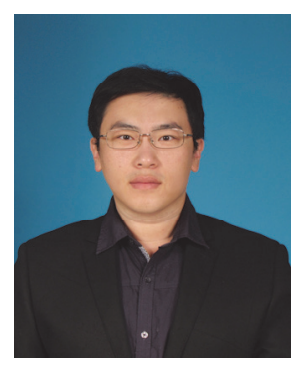

邱頔, 天津师范大学化学学院讲师. 2010 年于北京大学化 学与分子工程学院获得学士学位, 2015 年于北京大学化学与 分子工程学院获得理学博士学位, 有机化学专业, 师从王剑 波教授, 张艳教授. 2015 年进入天津师范大学化学学院工作. 目前主要从事芳香环的碳磷键构筑过程研究和芳香环基本官 能团的转化过程研究.

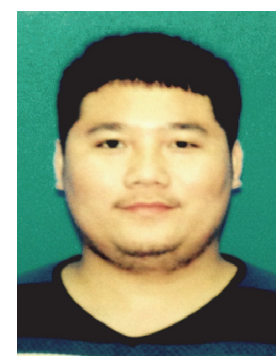

邱孟龙, 天津师范大学化学学院化学专业本科生. 2012 年 进入天津师范大学化学学院就读.

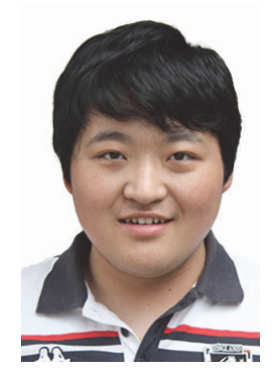

马戎, 天津师范大学化学学院化学专业本科生. 2012 年进 入天津师范大学化学学院就读.

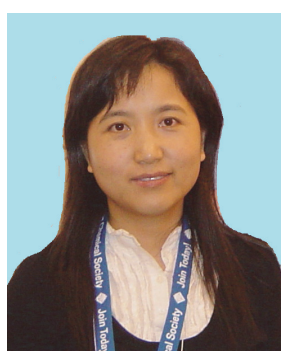

张艳, 北京大学化学与分子工程学院教授. 1997 年兰州大 学化学系本科毕业, 2002 年兰州大学化学系获博士学位(导师: 张自义教授). 随后分别于香港科技大学、奥地利因斯布鲁克 大学、德国莱比内茨研究所、美国密苏里大学和奥本大学从 事博士后及访问学者研究. 2008 年回国到北京大学化学与分 子工程学院任教. 主要从事有机合成反应研究, 在国内外主 要学术刊物发表论文 130 余篇, 出版译著 2 本.

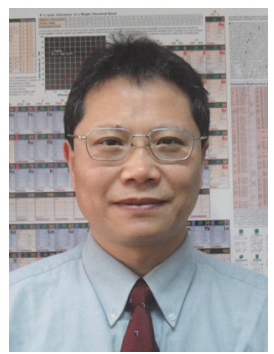

王剑波, 北京大学化学与分子工程学院教授, 生物有机 与分子工程教育部重点实验室主任. 1983 年南京理工大学本 科毕业, 1984 年国家教委公派留日学习, 1990 年于北海道大学 获工学博士学位(导师: Hiroshi Suginome 教授). 1990 1995 年 分别在瑞士日内瓦大学和美国威斯康星大学(麦迪逊)做博士 后. 1995 年底回国到北京大学化学学院任教. 2002 年获国家杰 出青年基金, 2006 年被聘为教育部长江特聘教授. 主要研究方 向为金属卡宾经由的催化反应. 独立工作以后在国内外主要 学术刊物发表论文 240 余篇.

\section{References}

[1] (a) Ye, T.; McKervey, M. A. Chem. Rev. 1994, 94, 1091; (b) Zhang, Z.; Wang, J. Tetrahedron 2008, 64, 6577; (c) Padwa, A.; Austin, D. J. Angew. Chem. Int. Ed. Engl. 1994, 33, 1797; (d) Padwa, A.; Weingarten, M. D. Chem. Rev. 1996, 96, 223; (e) Doyle, M. P.; Forbes, D. C. Chem. Rev. 1998, 98, 911; (f) Padwa, A. J. Organomet. Chem. 2001, 617-618, 3; (g) Davies, H. M. L.; Antoulinakis, E. G. J. Organomet. Chem. 2001, 617-618, 47. (h) Timmons, D. J.; Doyle, M. P. J. Organomet. Chem. 2001, 617-618, 98. (i) Hodgson, D. M.; Pierard, F. Y. T. M.; Stupple, P. A. Chem. Soc. Rev. 2001, 30, 50; (j) Davies, H. M. L.; Beckwith, R. E. J. Chem. Rev. 2003, 103, 
2861. (k) Singh, G. S.; Mdee, L. K. Curr. Org. Chem. 2003, 7, 1821. (1) Gois, P. M. P.; Afonso, C. A. M. Eur. J. Org. Chem. 2004, 3773. (m) Díaz-Requejo, M. M.; Pérez, P. J. J. Organomet. Chem. 2005, 690, 5441; (n) Fulton, J. R.; Aggarwal, V. K.; de Vicente, J. Eur. J. Org. Chem. 2005, 1479; (o) Davies, H. M. L.; Nikolai, J. Org. Biomol. Chem. 2005, 3, 4176. (p) Singh, G. S. Curr. Org. Synth. 2005, 2 , 377. (q) Wee, A. G. H. Curr. Org. Synth. 2006, 3, 499. (r) Noels, A. F. Angew. Chem. Int. Ed. 2007, 46, 1208.

[2] (a) Doyle, M. P.; McKervey, M. A.; Ye, T. Modern Catalytic Methods for Organic Synthesis with Diazo Compounds, Wiley-Interscience: New York, 1998. (b) For the recent comprehensive review of diazo compounds, see: Ford, A.; Miel, H.; Ring, A.; Slattery, C. N.; Maguire, A. R.; McKervey, M. A. Chem. Rev. 2015, 115,9981 .

[3] Maas, G. Angew. Chem. Int. Ed. 2009, 48, 8186.

[4] For typical research recently developed by Chinese chemists, see: (a) Cheng, Q.; Xu, H.; Zhu, S.; Zhou, Q. Acta Chim. Sinica 2015, 73, 326 (in Chinese). (程清卿, 许唤, 朱守非, 周其林, 化学学报, 2015, 73, 326.) (b) Tang, M.; Wu, Y.; Liu, Y.; Cai, M.; Xia, F.; Liu, S.; Hu, W. Acta Chim. Sinica 2016, 74, 54 (in Chinese). (唐敏, 吴 永, 刘源, 蔡茂强, 夏飞, 刘顺英, 胡文浩, 化学学报, 2016, 74, 54.) (c) Li, Y.; Huang, Z.; Xu, P.-F.; Zhang, Y.; Wang, J. Acta Chim. Sinica 2012, 70, 2024 (in Chinese). (李玉叶, 黄重行, 许鹏飞, 张 艳, 王剑波, 化学学报, 2012, 70, 2024.)

[5] Zhang, Y.; Wang, J. Chem. Commun. 2009, 5350.

[6] Hashimoto, T.; Maruoka, K. Bull. Chem. Soc. Jpn. 2013, 86, 1217.

[7] Yao, W.; Wang, J. Org. Lett. 2003, 5, 1527.

[8] (a) Arai, S.; Hasegawa, K.; Nishida, A. Tetrahedron Lett. 2004, 45, 1023. (b) Hasegawa, K.; Arai, S.; Nishida, A. Tetrahedron 2006, 62, 1390 .

[9] (a) Likhar, P. R.; Roy, S.; Roy, M.; Subhas, M. S.; Kantam, M. L. Synlett 2008, 1283. (b) Likhar, P. R.; Roy, S.; Roy, M.; Subhas, M. S.; Kantam, M. L. Catal. Commun. 2009, 10, 728.

[10] Trost, B. M.; Malhotra, S.; Koschker, P.; Ellerbrock, P. J. Am. Chem. Soc. 2012, 134, 2075.

[11] Uraguchi, D.; Sorimachi, K.; Terada, M. J. Am. Chem. Soc. 2005, $127,9360$.

[12] Hashimoto, T. ; Maruoka, K. J. Am. Chem. Soc. 2007, 129, 10054

[13] Hashimoto, T.; Maruoka, K. Synthesis 2008, 3703.

[14] Hashimoto, T.; Kimura, H.; Nakatsu, H.; Maruoka, K. J. Org. Chem. 2011, 76, 6030 .

[15] Doyle, M. P.; Kundu, K.; Russell, A. E. Org. Lett. 2005, 7, 5171.

[16] Kundu, K.; Doyle, M. P. Tetrahedron: Asymmetry 2006, 17, 574.

[17] Liu, Y.; Zhang, Y.; Jee, N.; Doyle, M. P. Org. Lett. 2008, 10, 1605.

[18] Zhou, L.; Doyle, M. P. Org. Lett. 2010, 12, 796.

[19] Xu, X.; Hu, W.-H.; Doyle, M. P. Angew. Chem. Int. Ed. 2011, 50, 6392.

[20] Mao, H.; Lin, A.; Shi, Y.; Mao, Z.; Zhu, X.; Li, W.; Hu, H.; Cheng, Y.; Zhu, C. Angew. Chem. Int. Ed. 2013, 52, 6288.

[21] Peng, C.; Cheng, J.; Wang, J. J. Am. Chem. Soc. 2007, 129, 8708.

[22] Ye, F.; Wang, C.; Zhang, Y.; Wang, J. Angew. Chem. Int. Ed. 2014, 53,11625 .

[23] (a) Rodriguez, J. B. Synthesis 2014, 46, 1129. (b) Dubrovskiy, A. V.; Markina, N. A.; Larock, R. C. Org. Biomol. Chem. 2013, 11, 191. (c) Hashimoto, T.; Maruoka, K. Org. Biomol. Chem. 2008, 6, 829.

[24] (a) Lozhkin, S. S.; Petrov, D. V.; Dokichev, V. A.; Tomilov, Y. V.; Nefedov, O. M. Chem. Heterocycl. Comp. 2009, 45, 937. (b) Novikov, R. A.; Platonov, D. N.; Dokichev, V. A.; Tomilov, Y. V.; Nefedov, O. M. Russ. Chem. Bull. Int. Ed. 2010, 59, 984. (c) Ovchinnikov, M. Y.; Yangirov, T. A.; Lobov, A. N.; Sultanova, R. M.; Khursan, S. L. Int. J. Chem. Kinet. 2013, 45, 499. (d) Krishna, P. R.; Sekhar, E. R.; Mongin, F. Tetrahedron Lett. 2008, 49, 6768; (e) Sun, H.; Wang, X.; Zhan, M.; Liu, J.; Xie, Y. Tetrahedron Lett. 2013, 54, 3846. (f) Wang, W.; Simovic, D. D.; Di, M.; Fieber, L.; Rein, K. S. Bioorg. Med. Chem. Lett. 2013, 23, 1949. (g) Ruano, J. L. G.; Alonso, M.; Cruz, D.; Fraile, A.; Martín, M. R.; Peromingo, M. T.; Tito, A.; Yuste, F. Tetrahedron 2008, 64, 10546. (h) Cruz, D. C.; Yuste, F.; Martín, M. R.; Tito, A.; Ruano, J. L. G. J. Org. Chem. 2009, 74, 3820. (i) Kissane, M.; Lawrence, S. E.; Maguire, A. R. Org. Biomol. Chem. 2010, 8, 2735. (j) Hamadi, N. B.; Msaddek, M. C. R. Chimie 2011, 14, 997. (k) Goulioukina, N. S.; Makukhin, N. N.; Beletskaya, I. P. Tetrahedron 2011, 67, 9535; (1) Liu, R.; Yin, J.; Li, J.; Wu, J.; Chen, G.; Jin, Y.; Wang, J. Chin. J. Org. Chem. 2012, 32, 544 (in Chinese). (刘再再, 殷军港, 李家柱, 武进, 陈冠龙, 金英学, 王进军, 有机化学, 2012, 32, 544.) (m) Yang, Z.; Wang,
Z.; Xu, X.; Liu, Y.; Qi, C.; Wang, J. Chin. J. Org. Chem. 2012, 32, 2099 (in Chinese). (杨泽, 王振, 徐希森, 刘洋, 祁彩霞, 王进军, 有机化学, 2012, 32, 2099.) (n) Xie, J.-W.; Wang, Z.; Yang, W.-J.; Kong, L.-C.; Xu, D.-C. Org. Biomol. Chem. 2009, 7, 4352; (o) Maurer, S.; Jikyo, T.; Maas, G. Eur. J. Org. Chem. 2009, 2195; (p) Hou, Y.; Cai, C.; Yu, G. Synlett 2016, 27, DOI: $10.1055 / \mathrm{s}-0035-1560596$

[25] (a) Gioiello, A.; Khamidullina, A.; Fulco, M. C.; Venturoni, F.; Zlotsky, S.; Pellicciari, R. Tetrahedron Lett. 2009, 50, 5978; (b) Wang, L.; Huang, J.; Gong, X.; Wang, J. Chem. Eur. J. 2013, 19, 7555 .

[26] (a) Slobodyanyuk, E. Y.; Artamonov, O. S.; Shishkin, O. V.; Mykhailiuk, P. K. Eur. J. Org. Chem. 2014, 2487; (b) Mykhailiuk, P. K. Chem. Eur. J. 2014, 20, 4942; (c) Artamonov, O. S.; Mykhailiuk, P. K.; Voievoda, N. M.; Volochnyuk, D. M.; Komarov, I. V. Synthesis 2010, 443; (d) Artamonov, O. S.; Slobodyanyuk, E. Y.; Shishkin, O. V.; Komarov, I. V.; Mykhailiuk, P. K. Synthesis 2013, 45, 225; (e) Li, T.-R.; Duan, S.-W.; Ding, W.; Liu, Y.-Y.; Chen, J.-R.; Lu, L.-Q.; Xiao, W.-J. J. Org. Chem. 2014, 79, 2296.

[27] (a) Muruganantham, R.; Namboothiri, I. J. Org. Chem. 2010, 75 2197; (b) Verma, D.; Mobin, S.; Namboothiri, I. N. N. J. Org. Chem. 2011, 76, 4764; (c) Kumar, R.; Namboothiri, I. N. N. Org. Lett. 2011, 13, 4016; (d) Kumar, R.; Nair, D.; Namboothiri, I. N. N. Tetrahedron 2014, 70, 1794; (e) Shelke, A. M.; Suryavanshi, G. Org. Biomol. Chem. 2015, 13, 8669.

[28] (a) Suga, H.; Furihata, Y.; Sakamoto, A.; Itoh, K.; Okumura, Y.; Tsuchida, T.; Kakehi, A.; Baba, T. J. Org. Chem. 2011, 76, 7377; (b) Gao, L.; Hwang, G.-S.; Lee, M. Y.; Ryu, D. H. Chem. Commun. 2009, 5460; (c) Lee, S. I.; Kim, K. E.; Hwang, G.-S.; Ryu, D. H. Org. Biomol. Chem. 2015, 13, 2745; (d) Du, T.; Du, F.; Ning, Y.; Peng, Y. Org. Lett. 2015, 17, 1308.

[29] (a) Mohanan, K.; Martin, A. R.; Toupet, L.; Smietana, M.; Vasseur, J.-J. Angew. Chem. Int. Ed. 2010, 49, 3196; (b) Martin, A. R.; Mohanan, K.; Toupet, L.; Vasseur, J.-J.; Smietana, M. Eur. J. Org. Chem. 2011, 3184.

[30] (a) He, S.; Chen, L.; Niu, Y.-N.; Wu, L.-Y.; Liang, Y.-M. Tetrahedron Lett. 2009, 50, 2443; (b) Cheung, K. M. J.; Reynisson, J.; McDonald, E. Tetrahedron Lett. 2010, 51, 5915; (c) McGrath, N. A.; Raines, R. T. Chem. Sci. 2012, 3, 3237. (d) Pramanik, M. M. D.; Kant, R.; Rastogi, N. Tetrahedron 2014, 70, 5214; (e) Vuluga, D.; Legros, J.; Crousse, B.; Bonnet-Delpon, D. Green Chem. 2009, 11, 156; (f) Friscourt, F.; Fahrni, C. J.; Boons, G.-J. Chem. Eur. J. 2015, 21, 13996.

[31] Li, F.; Nie, J.; Sun, L.; Zheng, Y.; Ma, J.-A. Angew. Chem. Int. Ed. 2013, 52, 6255 .

[32] (a) Mykhailiuk, P. K. Angew. Chem. Int. Ed. 2015, 54, 6558; (b) Mykhailiuk, P. K. Org. Biomol. Chem. 2015, 13, 3438; (c) Mykhailiuk, P. K. Eur. J. Org. Chem. 2015, 7235.

[33] (a) Shoji, Y.; Hari, Y.; Aoyama, T. Tetrahedron Lett. 2004, 45, 1769; (b) Jin, T.; Yamamoto, Y. Angew. Chem. Int. Ed. 2007, 46, 3387; (c) Liu, Z.; Shi, F.; Martinez, P. D. G.; Raminelli, C.; Larock, R. C. J. Org. Chem. 2008, 73, 219;. (d) Hari, Y.; Sone, R.; Aoyama, T. Org. Biomol. Chem. 2009, 7, 2804; (e) Wang, C.-D.; Liu, R.-S. Org. Biomol. Chem. 2012, 10, 8948; (f) Li, P.; Zhao, J.; Wu, C.; Larock, R. C.; Shi, F. Org. Lett. 2011, 13, 3340; (g) Li, P.; Wu, C.; Zhao, J.; Rogness, D. C.; Shi, F. J. Org. Chem. 2012, 77, 3149.

[34] (a) Pérez-Aguilar, M. C.; Valdés, C. Angew. Chem. Int. Ed. 2013, 52, 7219; (b) Sha, Q.; Wei, Y. Synthesis 2013, 45, 413; (c) Merchant, R. R.; Allwood, D. M.; Blakemore, D. C.; Ley, S. V. J. Org. Chem. 2014, 79, 8800; (d) Pérez-Aguilar, M. C.; Valdés, C. Angew. Chem. Int. Ed. 2015, 54, 13729.

[35] (a) Kang, T.; Kim, W.-Y.; Yoon, Y.; Kim, B. G.; Lee, H.-Y. J. Am. Chem. Soc. 2011, 133, 18050; (b) Qiao, Y.; Han, K.-L. Org. Biomol. Chem. 2014, 12, 1220; (c) Lee, H.-Y. Acc. Chem. Res. 2015, 48 , 2308.

[36] Zhang, F.-G.; Wei, Y.; Yi, Y.-P.; Nie, J.; Ma, J.-A. Org. Lett. 2014, 16,3122 .

[37] (a) Dullweber, F.; Montforts, F.-P. Synlett 2008, 3213; (b) Mlostoń, G.; Urbaniak, K.; Linden, A.; Heimgartner, H. Tetrahedron 2009, 65, 8191; (c) Assadi, N.; Pogodin, S.; Agranat, I. Eur. J. Org. Chem. 2011, 6773; (d) Nikolaev, V. A.; Ivanov, A. V.; Shakhmin, A. A.; Sieler, J.; Rodina, L. L. Tetrahedron Lett. 2012, 53, 3095; (e) Nikolaev, V. A.; Ivanov, A. V.; Rodina, L. L.; Mlostoń, G. Beilstein J. Org. Chem. 2013, 9, 2751.

[38] Torres-Alacan, J.; Sander, W. J. Org. Chem. 2008, 73, 7118. 
[39] Chen, J.-H.; Liu, S.-R.; Chen, K. Chem. Asian J. 2010, 5, 328.

[40] Chen, Z.; Fan, S.-Q.; Zheng, Y.; Ma, J.-A. Chem. Commun. 2015, 51,16545 .

[41] Wang, S.; Yang, L.-J.; Zeng, J.-L.; Zheng, Y.; Ma, J.-A. Org. Chem. Front. 2015, 2, 1468.

[42] (a) Sakač, M. N.; Gaković, A. R.; Csanádi, J. J.; Djurendić, E. A.; Klisurić, O.; Kojić, V.; Bogdanović, G.; Gaši, K. M. P. Tetrahedron Lett. 2009, 50, 4107. (b) Mani, N. S.; Fitzgerald, A. E. J. Org. Chem. 2014, 79, 8889 .

[43] (a) Supurgibekov, M. B.; Hennig, L.; Schulze, B.; Nikolaev, V. A. Russ. J. Org. Chem. 2008, 44, 1840; (b) Supurgibekov, M. B.; Zakharova, V. M.; Sieler, J.; Nikolaev, V. A. Tetrahedron Lett. 2011, 52, 341; (c) Muthusamy, S.; Srinivasan, P. Tetrahedron Lett. 2009, 50, 1331; (d) Bel Abed, H.; Mammoliti, O.; Van Lommen, G.; Herdewijn, P. Tetrahedron Lett. 2012, 53, 6489; (e) Bel Abed, H.; Mammoliti, O.; Bande, O.; Van Lommen, G.; Herdewijn, P. J. Org. Chem. 2013, 78, 7845; (f) Bel Abed, H.; Mammoliti, O.; Bande, O.; Van Lommen, G.; Herdewijn, P. Org. Biomol. Chem. 2014, 12, 7159; (g) Bel Abed, H.; Bande, O.; Mammoliti, O.; Van Lommen, G.; Herdewijn, P. Tetrahedron Lett. 2013, 54, 7056; (h) Nikolaev, V. A.; Cantillo, D.; Kappe, C. O.; Medvedev, J. J.; Prakash, G. K. S.; Supurgibekov, M. B. Chem. Eur. J. 2016, 22, 174.

[44] Mao, H.; Lin, A.; Tang, Z.; Hu, H.; Zhu, C.; Cheng, Y. Chem. Eur. J. 2014, 20, 2454.

[45] (a) Yasui, E.; Wada, M.; Takamura, N. Tetrahedron Lett. 2009, 50, 4762; (b) Yasui, E.; Wada, M.; Takamura, N. Tetrahedron 2009, 65, 461; (c) Yasui, E.; Wada, M.; Nagumo, S.; Takamura, N. Tetrahedron 2013, 69, 4325 .

[46] (a) van Berkel, S. S.; Brauch, S.; Gabriel, L.; Henze, M.; Stark, S.; Vasilev, D.; Wessjohann, L. A.; Abbas, M.; Westermann, B. Angew. Chem. Int. Ed. 2012, 51, 5343; (b) Kuznetsov, A.; Gulevich, A. V.; Wink, D. J.; Gevorgyan, V. Angew. Chem. Int. Ed. 2014, 53, 9021.

[47] Li, L.; Chen, J.-J.; Li, Y.-J.; Bu, X.-B.; Liu, Q.; Zhao, Y.-L. Angew. Chem. Int. Ed. 2015, 54, 12107.

[48] Li, W.; Liu, X.; Hao, X.; Hu, X.; Chu, Y.; Cao, W.; Qin, S.; Hu, C.; Lin, L.; Feng, X. J. Am. Chem. Soc. 2011, 133, 15268.
[49] (a) Santos, F. M. F.; Rosa, J. N.; André, V.; Duarte, M. T.; Veiros, L. F.; Gois, P. M. P. Org. Lett. 2013, 15, 1760; (b) António, J. P. M.; Frade, R. F. M.; Santos, F. M. F.; Coelho, J. A. S.; Afonso, C. A. M.; Gois, P. M. P.; Trindade, A. F. RSC Adv. 2014, 4, 29352.

[50] Mei, L.-Y.; Tang, X.-Y.; Shi, M. Chem. Eur. J. 2014, 20, 13136.

[51] Zheng, J.; Qi, J.; Cui, S. Org. Lett. 2016, 18, 128.

[52] Babinski, D. J.; Aguilar, H. R.; Still, R.; Frantz, D. E. J. Org. Chem. 2011, 76, 5915.

[53] Babinski, D. J.; Bao, X.; El Arba, M.; Chen, B.; Hrovat, D. A.; Borden, W. T.; Frantz, D. E. J. Am. Chem. Soc. 2012, 134, 16139.

[54] Guo, H.; Zhang, D.; Zhu, C.; Li, J.; Xu, G.; Sun, J. Org. Lett. 2014, 16,3110 .

[55] Xu, G.; Zhu, C.; Gu, W.; Li, J.; Sun, J. Angew. Chem. Int. Ed. 2015, 54,883 .

[56] Jordão, A. K.; Afonso, P. P.; Ferreira, V. F.; de Souza, M. C. B. V.; Almeida, M. C. B.; Beltrame, C. O.; Paiva, D. P.; Wardell, S. M. S. V.; Wardell, J. L.; Tiekink, E. R. T.; Damaso, C. R.; Cunha, A. C. Eur. J. Med. Chem. 2009, 44, 3777.

[57] Campos, V. R.; Abreu, P. A.; Castro, H. C.; Rodrigues, C. R.; Jordão, A. K.; Ferreira, V. F.; de Souza, M. C. B. V.; da C. Santos, F.; Moura L. A.; Domingos, T. S.; Carvalho, C. Sanchez, E. F.; Fuly, A. L.; Cunha, A. C. Bioorg. Med. Chem. 2009, 17, 7429.

[58] Wang, Z.; Bi, X.; Liao, P.; Zhang, R.; Liang, Y.; Dong, D. Chem. Commun. 2012, 48, 7076.

[59] Deng, G.; Wang, F.; Lu, S.; Cheng, B. Org. Lett. 2015, 17, 4651.

[60] Zhou, L.; Liu, Z.; Liu, Y.; Zhang, Y.; Wang, J. Tetrahedron 2013, 69, 6083.

[61] González, A.; Pérez, D.; Puig, C.; Ryder, H.; Sanahuja, J.; Solé, L.; Bach, J. Tetrahedron Lett. 2009, 50, 2750.

[62] Hasegawa, K.; Kimura, N.; Arai, S.; Nishida, A. J. Org. Chem. 2008, 73,6363 .

[63] Barluenga, J.; Lonzi, G.; Riesgo, L.; Tomás, M.; López, L. A. J. Am. Chem. Soc. 2011, 133, 18138.

[64] Qiu, L.; Huang, D.; Xu, G.; Dai, Z.; Sun, J. Org. Lett. 2015, 17, 1810 . 